\title{
Redox Activation of the Universally Conserved ATPase YchF by Thioredoxin 1
}

\author{
Liya Hannemann, Ida Suppanz,, 2,3 Qiaorui Ba, ${ }^{1,2}$ Katherine Maclnnes, \\ Friedel Drepper, ${ }^{2,3}$ Bettina Warscheid, ${ }^{2,3}$ and Hans-Georg Koch ${ }^{1}$
}

\begin{abstract}
Aims: YchF/Ola1 are unconventional members of the universally conserved GTPase family because they preferentially hydrolyze ATP rather than GTP. These ATPases have been associated with various cellular processes and pathologies, including DNA repair, tumorigenesis, and apoptosis. In particular, a possible role in regulating the oxidative stress response has been suggested for both bacterial and human YchF/Ola1. In this study, we analyzed how YchF responds to oxidative stress and how it potentially regulates the antioxidant response. Results: Our data identify a redox-regulated monomer-dimer equilibrium of YchF as a key event in the functional cycle of YchF. Upon oxidative stress, the oxidation of a conserved and surface-exposed cysteine residue promotes YchF dimerization, which is accompanied by inhibition of the ATPase activity. No dimers were observed in a YchF mutant lacking this cysteine. In vitro, the YchF dimer is dissociated by thioredoxin 1 (TrxA) and this stimulates the ATPase activity. The physiological significance of the YchF-thioredoxin 1 interaction was demonstrated by in vivo cross-linking, which validated this interaction in living cells. This approach also revealed that both the ATPase domain and the helical domain of $\mathrm{YchF}$ are in contact with TrxA. Innovation: YchF/Ola1 are the first redox-regulated members of the universally conserved GTPase family and are inactivated by oxidation of a conserved cysteine residue within the nucleotide-binding motif. Conclusion: Our data provide novel insights into the regulation of the so far ill-defined YchF/Ola1 family of proteins and stipulate their role as negative regulators of the oxidative stress response. Antioxid. Redox Signal. 24, 141-156.
\end{abstract}

\section{Introduction}

$\mathbf{P}$ -Loop GTPASEs are universally conserved molecular switches that cycle between a GDP-bound off state and a GTP-bound on state. The associated conformational changes then determine complex protein interaction networks that regulate essential processes such as protein synthesis, cellular differentiation, or stress response $(52,54)$. At least 20 GTPase families have been identified based on structural features and eight of these families are universally conserved in eukaryotes, bacteria, and archaea. Within the Obg (spoOBassociated GTP-binding protein) family of GTPases, the YchF/Ola1 proteins constitute an unconventional subfamily because they hydrolyze ATP rather than GTP $(24,49)$. This is due to an altered G4 motif in the nucleotide-binding domain,

\section{Innovation}

YchF/Ola1 represent largely uncharacterized ATPhydrolyzing members of the universally conserved GTPase family. Our data demonstrate that Escherichia coli YchF undergoes a redox-dependent dimerization. This inhibits its ATPase activity and prevents the inhibition of the antioxidant response. The monomer-dimer equilibrium of YchF as well as its ATPase activity is regulated by thioredoxin 1, which was identified by site-directed cross-linking as a major contact partner of $\mathrm{YchF}$ in vivo. Our data reveal a new regulatory mechanism for controlling the oxidative stress response and identify the YchF/Olal subfamily of $\mathrm{A}(\mathrm{G}) \mathrm{TPa}$ es as first redox-regulated members of the universally conserved GTPase family.

\footnotetext{
${ }^{1}$ Institut für Biochemie und Molekularbiologie, ${ }^{2}$ Faculty of Biology, and ${ }^{3}$ BIOSS Centre for Biological Signalling Studies, AlbertLudwigs-Universität Freiburg, Freiburg, Germany.
} 
which lacks a lysine residue that determines guanosine specificity (24).

The available crystal structures of Haemophilus influenzae YchF (47) and its human homologue hOla1 (24), show three distinct domains: the N-terminal domain displays a typical TRAFAC (translation factor)-type fold with a six-stranded mostly parallel $\beta$-sheet surrounded by $\alpha$-helices (Fig. 1A). The second domain is characterized by two $\alpha$-helices, which often serve as recognition motifs for protein and nucleic acid interaction (35). This YchF domain is similar to the coiledcoil domain in transcription elongation factor GreA (45), pointing to possible RNA interaction. The third domain is located at the C-terminus and shows similarity to TGS domains (ThrRS-GTPases-SpoT), which are associated with nucleotide-dependent regulatory functions and nucleic acid binding (2).

Despite its universal conservation, the physiological function of YchF/Ola1 is still unknown. Many Obg-like G/ ATPases are involved in ribosome biogenesis and ribosome binding has been observed for Escherichia coli $(6,49)$ and Trypanosoma cruzi (16) YchF. The exact impact of YchF on ribosome biogenesis or translation is unknown, but yeast Ola1 possibly influences translational fidelity (39). Whether YchF/Ola1 interact with ribosomal proteins or rRNA is unknown, but the cleft formed by the helical domain and the TGS domain would be wide enough to accommodate nucleic acids (Fig. 1A). A possible role in DNA repair was also postulated because human hOla1 is downregulated upon DNA damage and upregulated in many tumors (46). This was validated by the observation that hOlal interacts with BRCA1 (breast cancer-associated gene 1) at the centrosome and that depletion of hOla1 induced centrosome fragmentation (29). Other functions of Ola1/YchF include a possible role in iron uptake in pathogens $(8,15)$ and salinity stress in plants $(9,10)$.

The postulated functions of YchF/Ola1 in different species are difficult to reconcile with a single mode of action. However, in both $E$. coli and humans, overexpression of YchF/Ola1 resulted in $\mathrm{H}_{2} \mathrm{O}_{2}$ hypersensitivity $(53,56)$, suggesting a role of $\mathrm{YchF} / \mathrm{Ola} 1$ in oxidative stress response. This is so far the only predicted function of YchF/Ola1 that appears to be evolutionarily conserved from bacteria to humans. In E. coli, YchF was shown to interact with catalase KatG, the major $\mathrm{H}_{2} \mathrm{O}_{2}$-detoxifying enzyme, and overexpression of YchF decreased catalase activity in E. coli cell extracts (53). YchF/Ola1 probably act via a transcription- and translationindependent mechanism (56) because overexpression of YchF in E. coli had no significant influence on the OxyRcontrolled expression of KatG (53). OxyR is a LysR-like transcription factor, which serves as master regulator of the $\mathrm{H}_{2} \mathrm{O}_{2}$ response in E. coli (11). OxyR is activated by $\mathrm{H}_{2} \mathrm{O}_{2}$, resulting in increased expression of at least 30 proteins that either detoxify $\mathrm{H}_{2} \mathrm{O}_{2}$ or protect macromolecules against oxidative damage (19). A few genes are also repressed by oxidized OxyR; this includes $o x y R$ itself and, intriguingly, $y c h F$ $(19,53)$. This demonstrates that YchF is part of the E. coli OxyR regulon and probably acts as a negative regulator of the oxidative stress response. In addition, YchF appears to be regulated by complex post-translational modifications, including dephosphorylation of serine 16 (28) and so far unknown modifications, which are required for full ATPase activity in response to $\mathrm{H}_{2} \mathrm{O}_{2}(53)$.
In the current study, we have analyzed YchF in the model organism E. coli. Based on our data, we propose a model for the redox regulation of $\mathrm{YchF}$ and its inhibitory effect on the antioxidant response.

\section{Results}

\section{YchF forms redox-sensitive dimers}

The activity of $E$. coli $\mathrm{YchF}$ is regulated by an $\mathrm{H}_{2} \mathrm{O}_{2-}$ dependent dephosphorylation and additional, so far unknown, stress-dependent modifications (53). Redox-sensitive pathways are commonly regulated by either thiol modifications (3) or histidine oxidation (26). E. coli YchF contains six cysteine residues, which are highly conserved within enterobacteria and also weakly in other YchF/Ola1 homologues (Fig. 1B). In the structural model of E. coli YchF, which was generated using the $H$. influenzae YchF structure $(85 \%$ sequence identity to $E$. coli $\mathrm{YchF}$ ), the six cysteine residues are surface exposed (Fig. 1A), making them suitable for regulation via thiol oxidation.

To analyze this, YchF was purified from E. coli and separated by sodium dodecyl sulfate-polyacrylamide gel electrophoresis (SDS-PAGE) under reducing conditions (+25 mM DTT). Western blotting using $\alpha$-YchF antibodies detected YchF as a strong band at $\sim 40 \mathrm{kDa}$. In addition, a weak band at $\sim 90 \mathrm{kDa}$ was also detected, which could reflect a YchF dimer (Fig. 2A). Under nonreducing conditions (-DTT), the putative YchF dimer at $\sim 90 \mathrm{kDa}$ was much stronger and less of the monomer was detected (Fig. 2A). In addition, two weaker bands (*) of $\sim 50 \mathrm{kDa}$ and $100 \mathrm{kDa}$ were detected. The band migrating at $90 \mathrm{kDa}$ could, in principle, also correspond to a complex between YchF and additional proteins. To exclude this possibility, the $90 \mathrm{kDa}$ band was cut out of the gel and separated on SDS-PAGE under reducing conditions. Coomassie staining revealed a single band at $\sim 40 \mathrm{kDa}$ (Fig. 2B), demonstrating that the $90 \mathrm{kDa}$ band indeed corresponded to the YchF dimer.

Dimerization of purified $\mathrm{YchF}$ could be an artifact of the in vitro conditions and we therefore analyzed dimerization in cell extracts of E. coli cells expressing YchF under an arabinose-inducible promoter. Western blotting identified the YchF monomer and dimer in these crude cell extracts (Fig. 2C), demonstrating that dimerization is not only observed with purified proteins. We also tried to detect $\mathrm{YchF}$ and its dimeric form in wild-type $E$. coli cells, but the low cellular concentration of YchF and the sensitivity of our antibody did not allow for an unambiguous detection of the dimer in whole cells.

The dithiothreitol (DTT)-sensitive YchF dimerization supports the involvement of cysteine residues in dimerization and this was further analyzed by testing their surface accessibility using the thiol-modifying agent, methoxypolyethylene glycol maleimide (PEG-Mal). PEG-Mal binding to cysteines induces a mass shift that can be monitored by SDS-PAGE (7). When YchF was reduced by tris(2-carboxyethyl)phosphine (TCEP), incubated with PEG-Mal, and subsequently analyzed by SDSPAGE under reducing conditions, we observed seven prominent bands of increasing mass (Fig. 2D). As a control, we constructed and purified a cysteine-free YchF mutant. The Cys-free YchF did not show any mass shift upon PEG-Mal incubation (Fig. 2D). These data indicate that all six cysteine residues are accessible to PEG-Mal modification and thus are surface exposed as predicted from the homology model (Fig. 1A). 


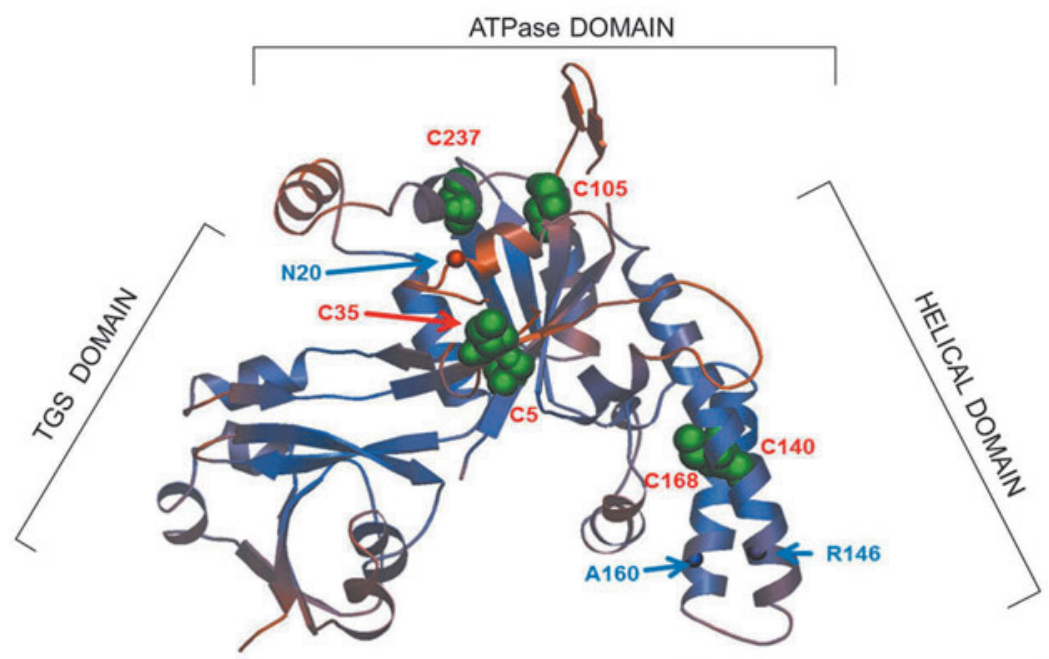

B

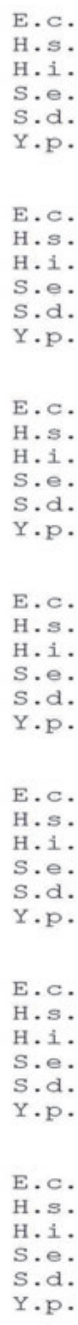

G1

G2

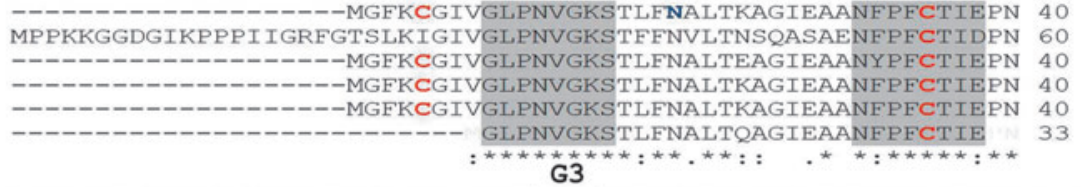

TGVVPMPDPRLDQLAEIVKPQRTLPTTMEFVDIAGLVKGASKGEGLGNQFLTNIRETEAI 100 ESRVPVPDERFDFLCQYHKPASKI PAFLNVVDIAGLVKGAHNGQGLGNAFLSHISACDGI 120 TGVVPMPDPRLDALAEIVKPERVLPTTMEFVDIAGLVAGASKGEGLGNKFLANIRETDAI 100 TGVVPMPDPRLDQLAEIVKPORILPTTMEFVDIAGLVKGASKGEGLGNOFLTNIRETEAI 100 TGVVPMPDPRLDQLAEIVKPQRTLPTTMEFVDIAGLVKGASKGEGLGNQFLTNIRETEAI 100

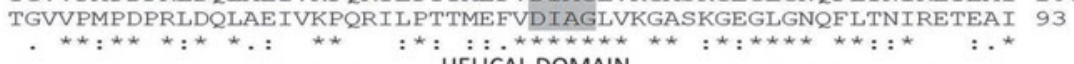
HELICAL DOMAIN

GHVVRCFENDNI IHVSGKVNPADDIEVINTELALADLDTCERAIHRVQKKAK-GGDKDAK 159 FHLTRAFEDDDITHVEGSVDPIRD IEI IHEELQLKDEEMIGPI IDKLEKVAVRGGDKKLK 180 GHVVRCFENDDIVHVAGKIDPLDDIDTINTELALADLDSCERAIORLOKRAK-GGDKEAK 159 GHVVRCFENDNI IHVAGKVNPAEDIDVINTEIAIADLDTCERAIHRVOKKAK-GGDKDAK 159 GHVVRCFENDNI I HVAGKVDPADDIETINTELALSDLETCERAIHRVQKKAK-GGDKDAK 152

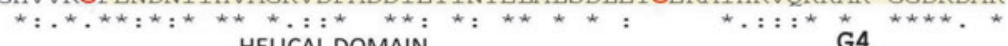
HELICAL DOMAIN G4

AELAVLEKCLPQLENAG--MLRALDLSAEEKAAIRYLSFLTLKPTMYIANVNEDGFE--N 215 PEYDIMCKVKSWVIDQKKPVRFYHDWNDKEIEVLNKHLFLTSKPMVYLVNLSEKDYIRKK 240 FELSVMEKILPVLENAG--MIRSVGLDKEELOA IKSYNFLTLKPTMYIANVNEDGFE--N 215 AELAALEKCLPHLAEAG--MLRSLDLTDEDKAAIRYLSFLTLKPTMYIANVNEDGFE--N 215 AELAVLEKCLPQLENAG--MLRALDLSAEEKAAIRYLSFLTLKPTMYIANVNEDGFE--N 215 AELEALEKCLPHLENAG--MLRALDLTAEDKAAIRYLSFLTLKPTMYIANVNEDGFE--N 208

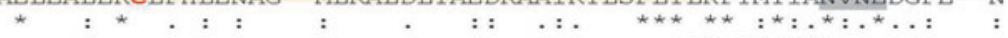
TGS- DOMAIN

NPYLDQVREIAAK--EGSVVVPVCAAVEADIAELDDEERDEFMQELGLEEPGLNRVIRAG 273 NKWLIKIKEWVDKYDPGALVIPFSGALELKLQELSAEERQKYLEAN-MTQSALPKIIKAG 299 NPYLDRVREIAAK--EGAVVVPVCAAIESEIAELDDEEKVEFLQDLGIEEPGLNRVIRAG 273 NPYLDQVREIAAK--EGSVVVPVCAAVEADIAELDDDERDEFMAELGLEEPGLNRVIRAG 273 NPYLDQVREIAAK--EGSVVVPVCAAVEADIAELDDEERDEFMOELGLEEPGLNRVIRAG 273 NPYLDQVRAIAAA--EGSVVVAVCAAVESDIAEMEDEDRAEFMAELGIEEPGLNRVIRAG 266

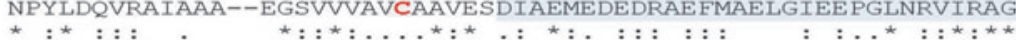
TGS- DOMAIN

YKLLNLQTYFTAGVKEVRAWTIPVGATAPQAAGKIHTDFEKGFIRAQTISFEDFITYKGE 333 FAALQLEYFFTAGPDEVRAWTIRKGTKAPQAAGKIHTDFEKGFIMAEVMKYEDFKEEGSE 359 YALLNLQTYFTAGVKEVRAWTVSVGATAPKAAAVIHTDFEKGFIRAEVIAYEDFIQFSGE 333 YRLLNLQTYFTAGVKEVRAWTI PVGATAPQAAGKI HTDFEKGFIRAQTIAFDDFITYKGE 333 YKLLNLOTYFTAGVKEVRAWTIPVGATAPQAAGKIHTDFEKGEIRAQTISFEDFITYKGE 333 YELLNLQTYFTAGVKEVRAWTIPVGATAPQAAGKIHTDFEKGFIRAQTIAFDDFITYKGE 326

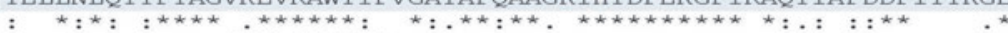
TGS- DOMAIN

QGAKEAGKMRAEGKDYIVKDGDVMNFLFNV------- 363 NAVKAAGKYRQQGRNYIVEDGDIIFFKFNTPQQPKKK 396 NGAKEAGKWRLEGKDYIVQDGDVMHFRFNV------- 363 QGAKEAGKMRAEGKDYIVKDGDIMNFLFNV------- 363 QGAKEAGKMRAEGKDYIVKDGDVMNFLFNV------- 363 QGAKTAGKMRSEGKEYIVKDGDVMNELFNV-

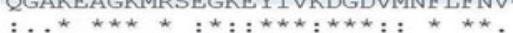

FIG. 1. Position and sequence conservation of cysteine residues in YchF/Ola1. (A) The crystal structure of the YchF homologue of Haemophilus influenzae (1JAL) was used to model the probable position of the six cysteine residues in Escherichia coli YchF using the Swiss Model (http://swissmodel.expasy.org/interactive/kFyFA3/models). Cysteine residues are labeled with green spheres and the amino acid position based on the E. coli numbering is indicated in red. In addition, the residues where the UV-induced cross-linker pBpa was incorporated are indicated by circles and the amino acid position based on the E. coli numbering is indicated in blue. (B) A sequence alignment of YchF homologues from different enterobacteria was generated by using the Clustal 2.0 software and compared with the $H$. influenzae YchF and Homo sapiens Ola1 sequence. Cysteine residues are shown in red and bold and the positions where pBpa was incorporated in the E. coli YchF are shown in blue and bold. The G1-G4 motifs of the nucleotide-binding site are boxed in gray, the helical domain is boxed in yellow, and the TGS domain in blue. E.c., Escherichia coli; H.s., Homo sapiens; H.i., Haemophilus influenzae; S.e., Salmonella enterica; Y.p., Yersinia pestis; S.d., Shigella dysentericae. To see this illustration in color, the reader is referred to the web version of this article at www.liebertpub.com/ars 
A

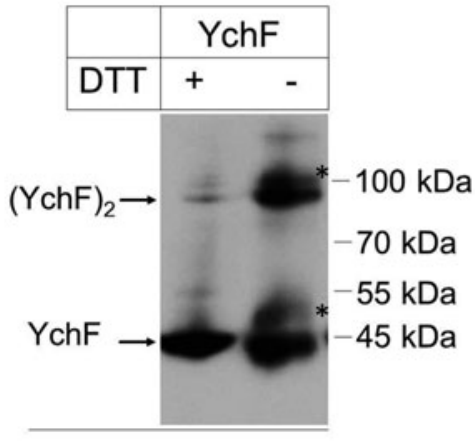

C

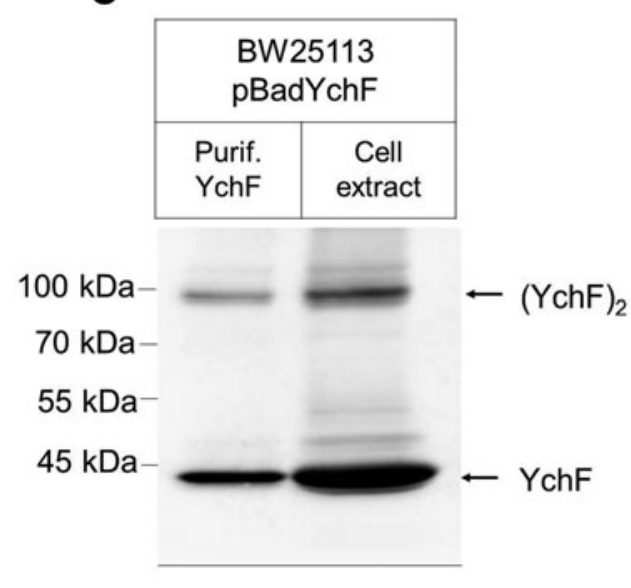

E

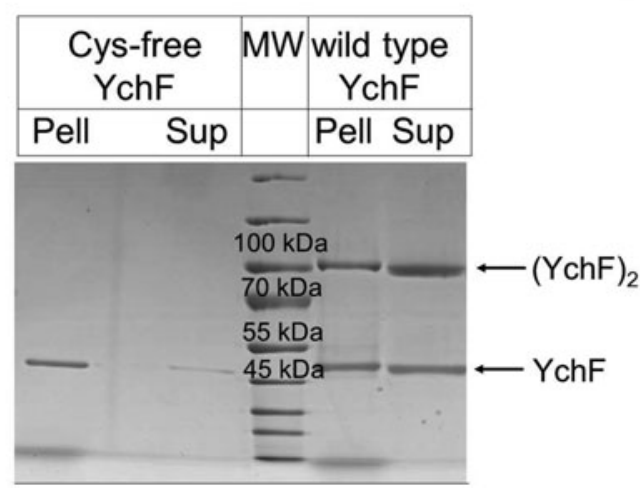

B

D
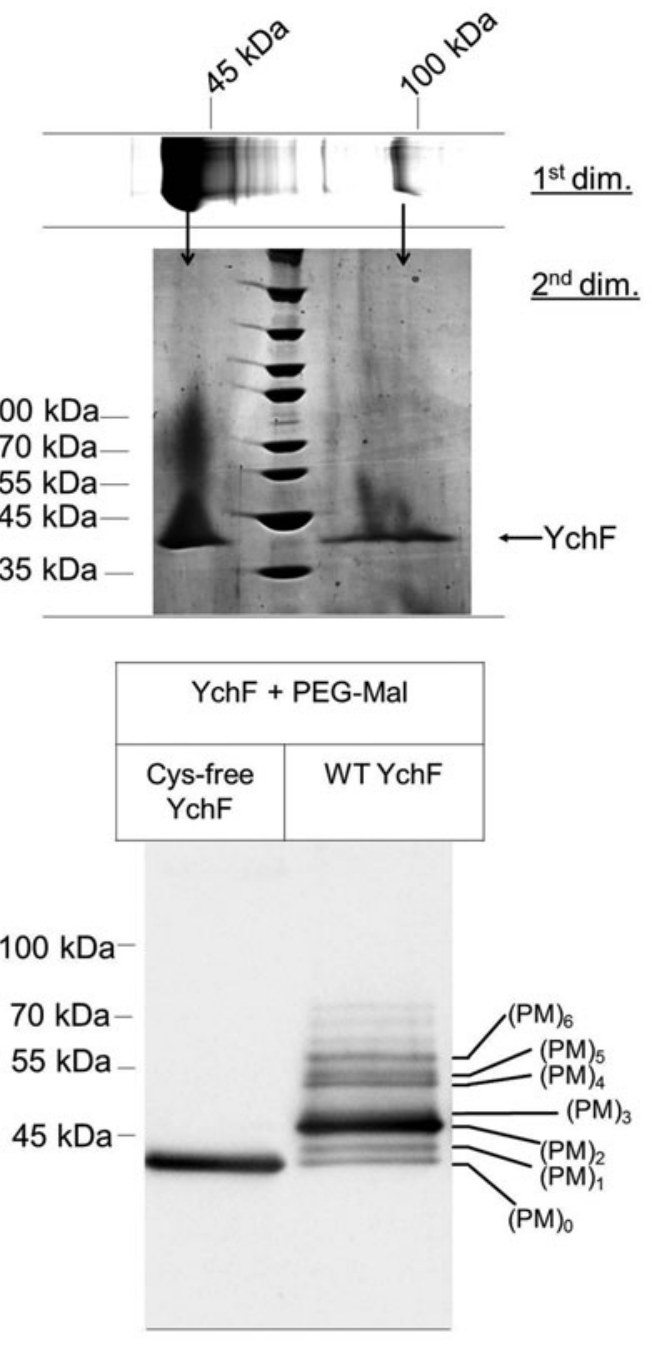

F

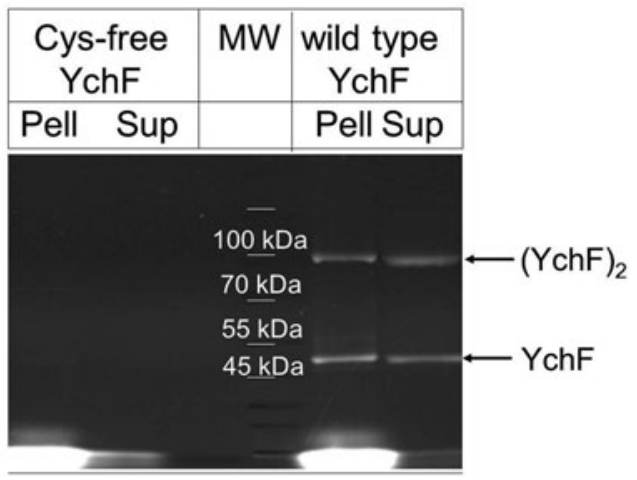

FIG. 2. YchF forms redox- and cysteine-dependent physiological dimers. (A) Purified YchF was denatured at $37^{\circ} \mathrm{C}$ for $15 \mathrm{~min}$ at either reducing (+25 mM DTT) or nonreducing conditions (-DTT) and after sodium dodecyl sulfate-polyacrylamide gel electrophoresis (SDS-PAGE) and Western transfer probed with $\alpha$-YchF antibodies. Two weaker bands were also recognized by $\alpha$-YchF antibodies (*) in the absence of DTT. (B) Purified YchF was separated on SDS-PAGE under nonreducing conditions as in A (1st dim.), and after Coomassie staining, the YchF monomer and dimer bands were excised out of the gel and separated on a second dimension SDS-PAGE (2nd dim.) under reducing conditions. (C) Purified YchF and lysozyme-lysed E. coli cells expressing YchF were separated under nonreducing conditions on SDS-PAGE and YchF was detected after Western transfer with $\alpha-Y c h F$ antibodies. (D) Purified wild-type YchF (wt YchF) and a cysteine-free derivative (Cys-free YchF) were reduced and incubated with the thiol-modifying agent, PEG-Mal, and subsequently separated by SDS-PAGE. After Western transfer, the membrane was probed with $\alpha$-YchF antibodies. $(\mathrm{PM})_{0-6}$ reflect the number of labeled cysteines. $(\mathbf{E}, \mathbf{F})$ Purified Cys-free and wild-type YchF were incubated with the fluorescent thiol-modifying agent fluorescein-5-maleimide. Subsequently, pellet (Pell) and supernatant (Sup) fractions were prepared and samples separated by SDS-PAGE under nonreducing conditions, followed by Coomassie staining (E) or in-gel fluorescence analysis (F). 
The contribution of the cysteine residues to YchF dimerization was further analyzed by comparing wild-type YchF and the Cys-free YchF mutant by SDS-PAGE under nonreducing conditions. Cys-free YchF showed a tendency to aggregate and purified proteins were therefore subjected to a centrifugation step before SDS-PAGE. For wild-type YchF, we observed both monomer and dimer by Coomassie staining (Fig. 2E). Both forms were almost equally distributed between the supernatant and pellet fraction after centrifugation. For the Cys-free mutant, we observed only the monomeric form, but no dimer (Fig. 2E), which demonstrates that YchF dimerization is cysteine dependent. The Cys-free YchF monomer was predominantly recovered from the pellet fraction after centrifugation, indicating that the cysteine residues are important for folding or solubility of YchF. Dimerization was also analyzed by the cysteine-specific fluorescent dye fluorescein-5-maleimide, which stained both monomer and dimer of wild-type YchF, but not the Cys-free mutant (Fig. 2F). This verifies the absence of cysteine residues in the Cys-free $\mathrm{YchF}$ derivative and indicates that not all cysteine residues of wild-type $\mathrm{YchF}$ are involved in dimerization. Cys-free $\mathrm{YchF}$ was purified under denaturing conditions and subsequently refolded. For excluding the possibility that the absence of the dimer observed for the Cys-free $\mathrm{YchF}$ is the result of the purification procedure, we also purified wild-type YchF under denaturing conditions and found both the monomeric and dimeric forms (Supplementary Fig. S1; Supplementary Data are available online at www.liebertpub.com/ars).

\section{The conserved cysteine residue 35 is required} for YchF dimerization

For identifying the cysteine residues involved in YchF dimerization, purified YchF was treated with iodoacetamide for blocking free cysteine residues and separated by SDSPAGE. After colloidal Coomassie staining, the monomer and dimer bands were excised and analyzed by high-resolution mass spectrometry (MS). This approach identified two uniquely cysteine-linked peptides (Table 1 and Fig. 3). In the YchF monomer, peptides containing Cys5 and Cys 35 formed a prominent intramolecular disulfide bridge, which was virtually absent in the YchF dimer (Fig. 3A). Instead, Cys35Cys35-linked peptides were present in the dimer and not in the monomer (Fig. 3B, C). This demonstrates that YchF dimerizes via disulfide bridge formation between the Cys35 residues of each monomer, whereas in the monomeric YchF, Cys5 forms an intramolecular disulfide bond to Cys35.

The involvement of Cys35 in YchF dimerization was further verified by analyzing dimer formation in a $Y \operatorname{chF}$ mutant that contained a cysteine 35-to-serine replacement. In this mutant, almost no YchF dimer was detectable by Coomassie staining or Western blotting (Fig. 3D, E). This confirms that Cys35 is essential for dimer formation. For wild-type $\mathrm{YchF}$ and the $\mathrm{YchF}(\mathrm{C} 35 \mathrm{~S})$ mutant, an additional band at $\sim 50 \mathrm{kDa}$ was detected, suggesting a further Cys35independent form of YchF. Using MS, we confirmed that YchF is the main component $(99.5 \%)$ in this band. As minor contamination $(0.5 \%)$, we only detected 3-dehydroquinate synthase (AroB), which has a molecular mass of $39 \mathrm{kDa}$ and is probably copurifying with YchF. Thus, further analysis is needed to reveal the identity of this Cys35-independent 50$\mathrm{kDa}$ YchF form.

Intriguingly, among the cysteine residues present in different YchF/Ola1 species, Cys35 shows the highest conservation (Fig. 1B). This may indicate that Cys35-linked dimer formation is a general feature of the $\mathrm{YchF} / \mathrm{Ola} 1$ protein family.

\section{YchF displays redox-activated ATPase activity}

To determine the physiological significance of the redoxdependent dimerization, we monitored the ATPase activity of YchF. We first measured the ATPase activity of purified wildtype YchF under reducing (+DTT) and oxidizing conditions (+tetrathionate). The addition of DTT stimulated the ATPase activity of YchF about two-fold, while the addition of tetrathionate significantly reduced the ATPase activity (Fig. 4A).

We then compared the ATPase activity of wild-type YchF with the activity of the $\mathrm{YchF}(\mathrm{C} 35 \mathrm{~S})$ mutant and the Cys-free YchF. In both the C35S and the Cys-free mutant, we detected an approximately two-fold higher ATPase activity than in the wild type. The addition of DTT had no significant effect on the Cys-free or the C35S YchF mutants (Fig. 4B). In summary, these data demonstrate that the ATPase activity of YchF is determined by its redox-sensitive dimerization. The YchF dimer exhibits only low activity, but the dissociation into the monomer strongly stimulates its ATPase activity.

The overexpression of YchF/Ola1 in E. coli or humans increases the sensitivity toward oxidative stress $(53,56)$. This phenotype depends on the ATPase activity of $\mathrm{YchF}(53)$ and we therefore reasoned that expressing the $\mathrm{YchF}(\mathrm{C} 35 \mathrm{~S}) \mathrm{mu}-$ tant should result in enhanced oxidative stress sensitivity,

Table 1. Disulfide-Linked Peptides of YchF Determined by Liquid Chromatography-Tandem MASS SPECTROMETRY

\begin{tabular}{|c|c|c|c|c|c|c|}
\hline Peptide A sequence & Peptide B sequence & $\begin{array}{c}\text { Mass } \\
(M r) / D a\end{array}$ & $\begin{array}{l}\text { Charge } \\
\text { state }\end{array}$ & $\begin{array}{l}\text { Identified } \\
\quad \text { in }\end{array}$ & $\begin{array}{l}\text { Area } \\
\text { dimer }\end{array}$ & $\begin{array}{c}\text { Area }^{\mathrm{a}} \\
\text { monome }\end{array}$ \\
\hline$C^{5}$ GIVGLPNVGK & AGIEAANFPFC ${ }^{35}$ TIEPNTGVVPM*PDPR & 3811.8703 & 3,4 & Monomer & $1.5 \mathrm{E} 7$ & $3.9 \mathrm{E} 8$ \\
\hline $\begin{array}{l}\text { AGIEAANFPFC }{ }^{35} \\
\text { TIEPNTGVVPM*PDPR }\end{array}$ & AGIEAANFPFC ${ }^{35}$ TIEPNTGVVPM*PDPR & 5514.6123 & 4 & Dimer & $1.5 \mathrm{E} 8$ & $5.9 \mathrm{E} 6$ \\
\hline $\begin{array}{l}\text { AGIEAANFPFC }{ }^{35} \\
\text { TIEPNTGVVPMPDPR }\end{array}$ & AGIEAANFPFC ${ }^{35}$ TIEPNTGVVPM*PDPR & 5498.6171 & 4 & Dimer & 4.6E7 & n. d. \\
\hline
\end{tabular}

Monomer and dimer bands were excised from the polyacrylamide gel and subjected to in-gel digestion using trypsin, followed by LCMS/MS analysis. For the identification of disulfide-linked peptides, mass spectrometric data were analyzed using the xQuest/xProphet software suite. Quantitative data analysis was performed with the Skyline software.

${ }^{\mathrm{a}}$ chromatographic peak area summed for molecular ion MS1 isotope peaks.

$\mathrm{M}^{*}$, oxidized methionine; n.d., not detected. 

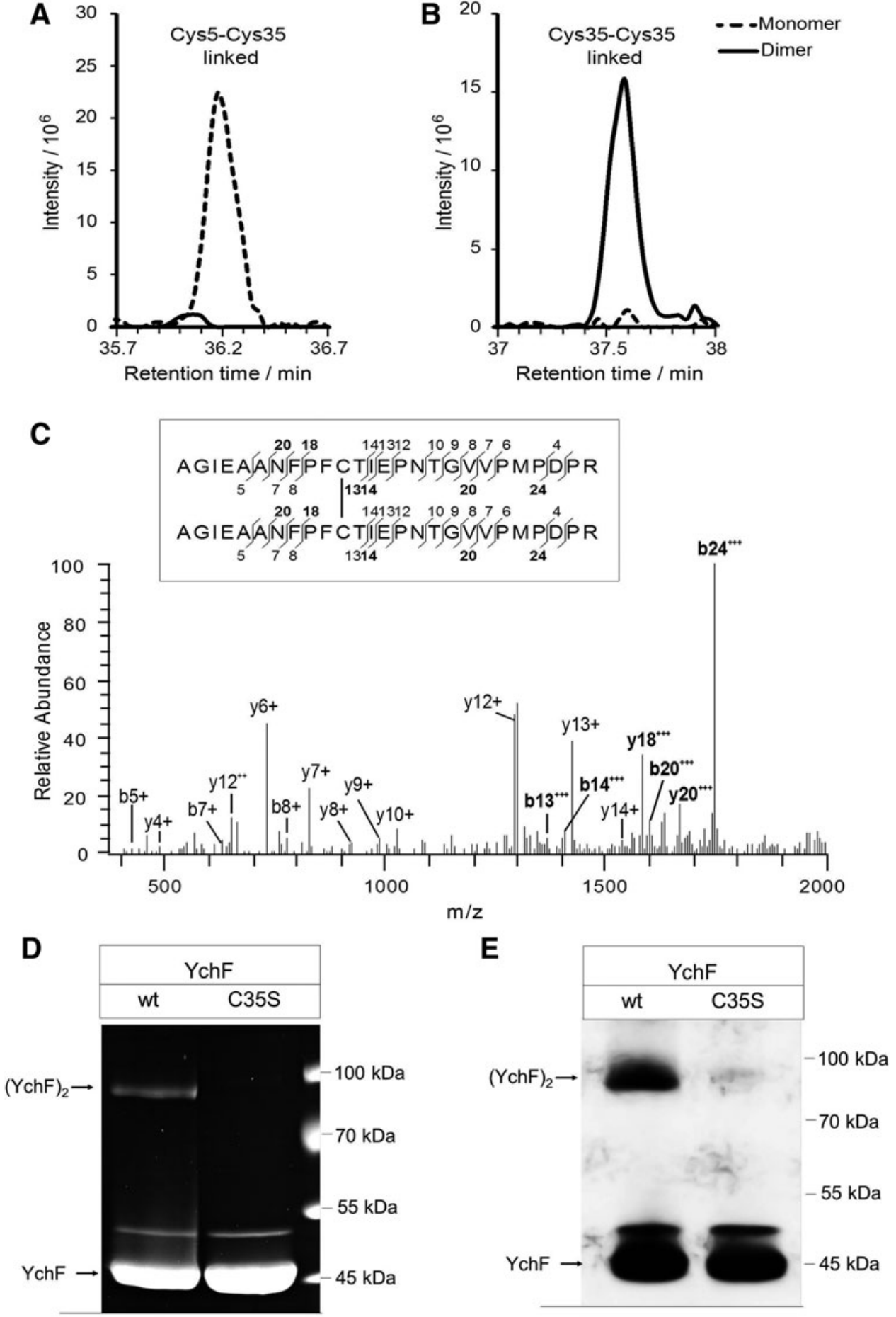

FIG. 3. Identification and quantification of disulfidelinked peptides in YchF monomer and dimer. (A) Extracted ion chromatograms of the Cys5-Cys35-linked peptides, CGIVGLPNVGK and AGIEAANFPFCTIEPNTGV VPM*PDPR (precursor $\mathrm{m} / \mathrm{z}$ 953.9757, charge +4), determined by liquid chromatography-mass spectrometry analyses of the monomer (dashed line) and dimer band (solid line). (B) Equivalent to A, but for the Cys35-Cys35-linked peptide AGIEAANFPFCTIEPNTGV VPM*PDPR (precursor $\mathrm{m} / \mathrm{z}$ 1379.6581, charge +4). (C) Fragment spectrum of the Cys35-Cys35 disulfide-linked peptide AGIEAANFPFCTIEP NTGVVPM*PDPR. b- and ytype fragment ions are annotated in the mass spectrum and in the sequence (inset). Fragments containing the disulfide bridge are shown in bold. (D) Wild-type YchF and YchF containing a single cysteine-toserine replacement at position 35 (YchFC35S) were purified and separated by SDS-PAGE under nonreducing conditions. Proteins were subsequently stained with Coomassie. (E) As in $\mathbf{D}$, but samples were probed with $\alpha-Y c h F$ antibodies after Western blotting. $\mathrm{M}^{*}$, oxidized methionine. which was induced by the thiol-modifying agent, diamide. Diamide induces reversible non-native disulfide bonds in cytoplasmic proteins (19), thereby reducing the free thiol concentration. Diamide sensitivity was analyzed by agar diffusion assays (Fig. 4C) and by spotting serially diluted cells on diamide-containing plates (Fig. 4D). Both assays demonstrated increased sensitivity of the $\mathrm{YchF}(\mathrm{C} 35 \mathrm{~S})$ expressing $\triangle y c h F$ strain compared with the $\triangle y c h F$ strain expressing wild-type $\mathrm{YchF}$ or the plasmid-free $\Delta y c h F$ strain (Fig. 4C, D). Expressing the ATPase-deficient YchF(P11A/
N12A) derivative, which shows reduced ATPase activity (53), had only a minor effect on diamide sensitivity (Fig. 4C). These data indicate that the high ATPase activity of the $Y \operatorname{chF}(\mathrm{C} 35 \mathrm{~S})$ mutant inhibits the ability of $E$. coli to cope with oxidative stress.

\section{YchF interacts with thioredoxin 1 in vivo}

The redox-sensitive monomer-dimer transition of YchF and its effect on the ATPase activity raised the question about 
A

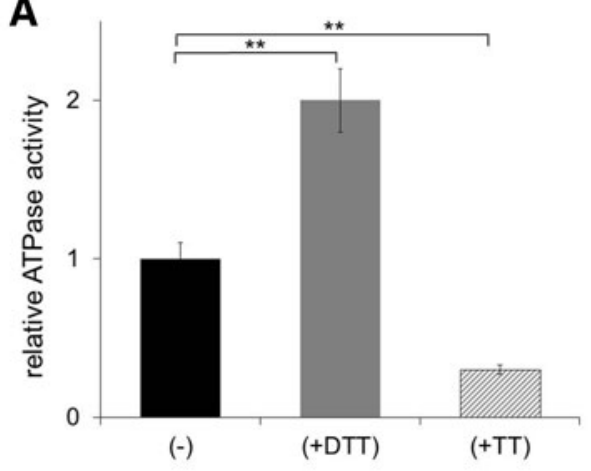

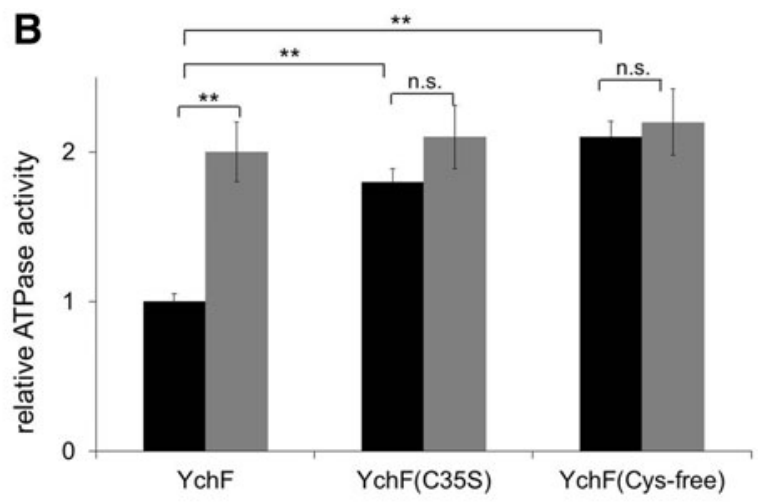
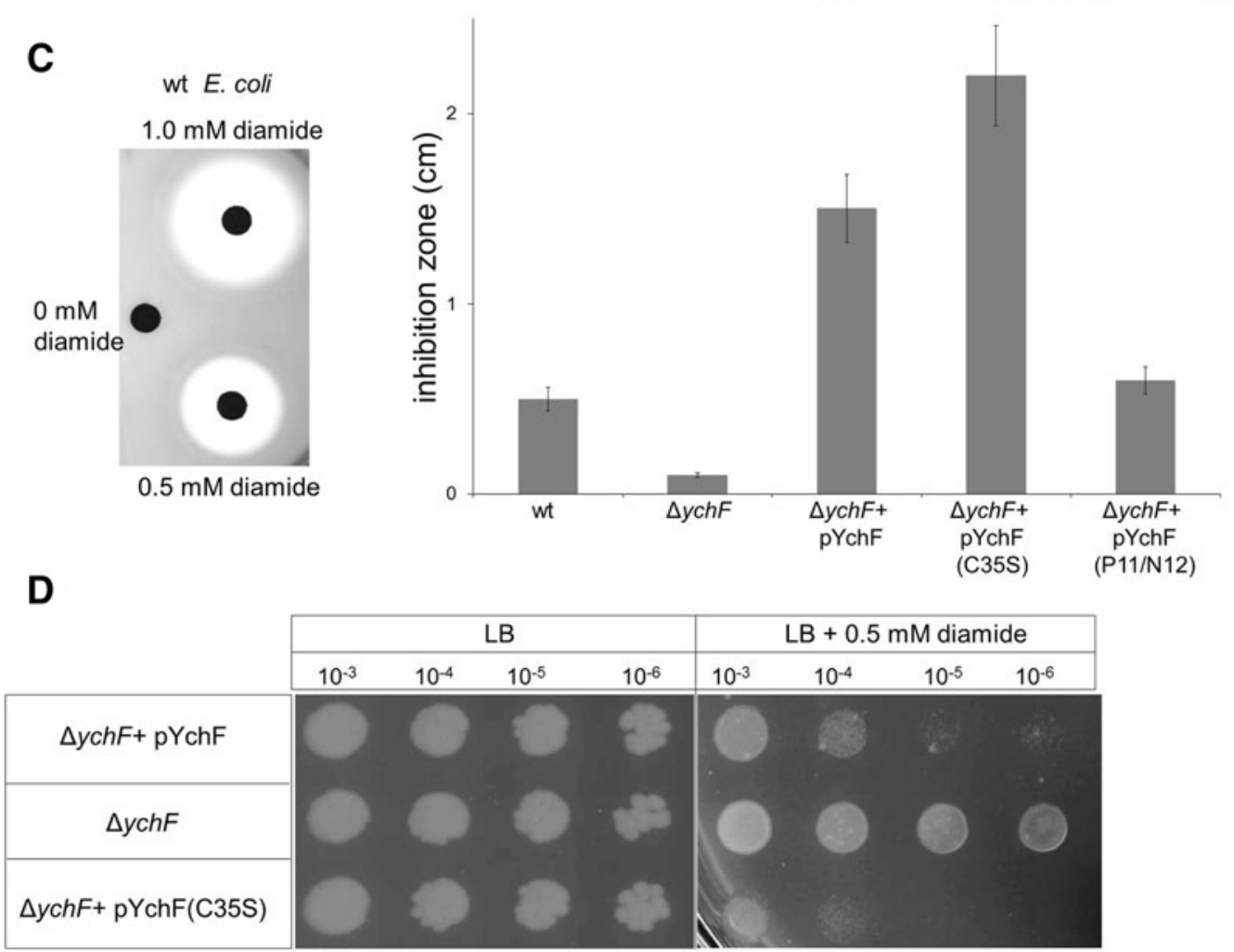

FIG. 4. Dimerization of YchF inhibits its ATPase activity (A) The ATPase activity of purified wild-type YchF (black bar) was determined by measuring phosphate release of $\gamma_{-}^{33} \mathrm{P}$-labeled ATP. When indicated, YchF was preincubated with either $10 \mathrm{mM}$ DTT (gray bar) or $10 \mathrm{~m} M$ tetrathionate (TT) (hatched bar) for $5 \mathrm{~min}$ at room temperature before measuring the activity. A relative YchF activity of 1 corresponds to $\sim 0.35 \mathrm{nmol} \mathrm{ATP} /(\min \times \mathrm{mg}$ protein). The relative ATPase activities shown are the mean $\pm \mathrm{SD}(n>3) . * * p<0.01$. (B) The ATPase activities of wild-type YchF, the $\mathrm{YchF}(\mathrm{C} 35 \mathrm{~S})$ mutant, and of the cysteine-free YchF mutant were determined without (black bars) or after preincubation with $10 \mathrm{~m} M$ DTT (gray bars). A relative YchF activity of 1 corresponds to $\sim 0.35 \mathrm{nmol} \mathrm{ATP} /(\mathrm{min} \times \mathrm{mg}$ protein). The relative ATPase activities shown are the mean $\pm \mathrm{SD}$ $(n=3)$. $* * p<0.01$; n.s. not significant. (C) E. coli strains were adjusted to an optical density of 0.5 , mixed with Top agar, and poured on LB Plates. Filter discs were soaked with different diamide concentrations and placed on these plates. Inhibition zones were quantified after $\sim 5 \mathrm{~h}$ of growth and the results for wild-type E. coli are shown (left panel). Quantification of two independent experiments in the presence of $0.5 \mathrm{~m} M$ diamide (right panel). $\mathrm{pYchF}(\mathrm{P} 11 / \mathrm{N} 12)$ corresponds to an ATPasedeficient YchF derivative. (D) The indicated E. coli strains were grown overnight on liquid LB medium without antibiotics and adjusted to an optical density of 1.0 before serial dilution. Dilutions were then spotted onto LB plates or LB plates containing $0.5 \mathrm{~m} M$ diamide. Plates were incubated at $37^{\circ} \mathrm{C}$ and analyzed after $\sim 12 \mathrm{~h}$ of growth.

the physiological redox partner proteins of YchF. This was addressed by using a site-directed in vivo photo-cross-linking approach with para-benzoyl-L-phenylalanine (pBpa). pBpa can be incorporated specifically at amber stop codon positions in the presence of a plasmid-borne orthogonal aminoacyl-tRNA synthetase/tRNA $\mathrm{CUA}_{\mathrm{A}}$ pair (37). pBpa was first incorporated into position 20 of YchF. Position 20 is located between cysteine residues, Cys5 and Cys35, and had been cross-linked to catalase KatG (53). E. coli cells expressing pBad-YchF(N20pBpa) were UV exposed to induce the cross-link reaction in vivo and $\mathrm{YchF}$ and its cross-linked partner proteins were then purified. As a control, we analyzed 
samples without UV exposure and samples from cells expressing pBad-YchF without pBpa. Following SDS-PAGE and MS analysis of cross-linked samples and the respective controls, we analyzed the data specifically for cross-links between $\mathrm{YchF}$ and proteins that are either associated with the oxidative stress response or involved in maintaining redox homeostasis. In support of previous data (53), we found the E. coli catalases, KatG and KatE, as well as the large subunit of the alkyl hydroperoxide reductase AhpF cross-linked to YchF at position 20 (Table 2). KatG, KatE, and AhpF were also identified in the purified YchF sample without UV exposure (Table 2), supporting the previously observed copurification of these proteins together with YchF. When searching the MS data for potential YchF cross-links to proteins controlling redox homeostasis, we found strong cross-links to thioredoxin 1 (TrxA) and weaker cross-links to thioredoxin 2 (TrxC), peroxiredoxin, and glutaredoxin-4 (Table 2).

The strong cross-link between YchF and TrxA was further verified by immune detection. When $\mathrm{YchF}$ was purified from UV-exposed E. coli pBad-YchF(N20pBpa) cells, we observed a strong UV-dependent band at $\sim 50 \mathrm{kDa}$, which was recognized by $\alpha$-TrxA antibodies (Fig. 5A). This band was absent when $\mathrm{pBad}-\mathrm{YchF}(\mathrm{N} 20 \mathrm{pBpa})$ was expressed in a $\Delta$ trx $A$ knockout strain. These data demonstrate that YchF interacts with TrxA in vivo. We also noticed that TrxA copurified with YchF because it was detected as an $\sim 10 \mathrm{kDa}$ band in the purified YchF sample (Fig. 5A). Upon UV exposure, the copurifying TrxA amount became significantly weaker, presumably because most of TrxA was cross-linked to YchF (Fig. 5A). To analyze the contribution of the cysteine residues of YchF to the TrxA interaction, we reduced the concentration of available thiol groups by treating the cells before crosslinking with a low concentration of diamide. At concentrations below $100 \mu M$, diamide does not induce the OxyR response (58) and, in agreement with this, the diamide-treated sample did not show significantly changed YchF or TrxA levels (Fig. 5B). However, upon diamide treatment, we observed a significant reduction in the YchF-TrxA cross-linking efficiency (Fig. 5C). We also analyzed the cross-link between YchF and peroxiredoxin and found reduced amounts of the cross-linked product upon diamide treatment (Fig. 5D). Thus, decreasing the number of available thiol groups impairs the interaction of YchF with TrxA and peroxiredoxin, which suggests that the cysteine residues of $\mathrm{YchF}$ are important for these interactions.

The specificity of the YchF-TrxA interaction was further analyzed by incorporating pBpa into positions 146 and 160 of YchF. These residues are close to Cys140 and Cys168 and located within a predicted coiled-coil structure of YchF. Western blotting showed YchF-TrxA cross-links for both positions (Fig. 6), which were confirmed by MS. However, the cross-linked products from positions 146 and 160 appeared weaker than the cross-link from the N20 residue. Furthermore, both cross-linked products migrated as a double band; a weaker band migrating at $\sim 50 \mathrm{kDa}$, and a stronger band migrating at $\sim 55 \mathrm{kDa}$. Position-dependent mobility of pBpa cross-linked products on SDS-PAGE is frequently observed $(25,31,38)$ and probably the result of differences in the three-dimensional structures of the cross-linking products. We also noticed less TrxA copurifying with YchF that had pBpa inserted into positions 140 and 160, which could indicate that $\mathrm{pBpa}$ insertion at these residues reduces the YchF-TrxA interaction.

In summary, our data show that the N-terminus of YchF and the predicted coiled-coil domain are cross-linked to TrxA. Reducing the concentration of free thiol groups by diamide treatment reduces the YchF-TrxA cross-linking efficiency, suggesting that the YchF-TrxA interaction probably involves mixed disulfides.

\section{Thioredoxin 1 activates the ATPase activity of YchF by dimer dissociation}

The physiological significance of the YchF-TrxA interaction was determined by analyzing the ATPase activity of YchF in the presence or absence of TrxA. TrxA was added to purified YchF and the ATPase activity was measured. In the

Table 2. YchF Interacts with Proteins of the Antioxidant Response

\begin{tabular}{|c|c|c|c|c|c|}
\hline \multirow[b]{2}{*}{ Protein $^{\mathrm{a}}$} & \multicolumn{5}{|c|}{$Y \operatorname{chF}(N 20 p B p a)$} \\
\hline & $\begin{array}{l}\text { Mol. Mass b } \\
\quad(k D a)\end{array}$ & $\begin{array}{c}\text { Gel Mol. } \\
\operatorname{Mass}^{\mathrm{c}}(k D a)\end{array}$ & $\begin{array}{l}\text { Rel. Intensity }^{\mathrm{d}} \\
(-U V /+U V)\end{array}$ & $\begin{array}{c}\text { Coverage } \\
(\%)\end{array}$ & Peptides $^{\mathrm{f}}$ \\
\hline Catalase G (KatG) & 80 & 120 & 0.6 & 68 & 70 \\
\hline Catalase E (KatE) & 84 & 120 & 0.3 & 9 & 6 \\
\hline Alkyl peroxidase subunit $\mathrm{F}(\mathrm{AhpF})$ & 56 & 95 & 0.7 & 78 & 56 \\
\hline Thioredoxin 1 (TrxA) & 12 & 55 & 0.001 & 78 & 8 \\
\hline Thioredoxin 2 (TrxC) & 16 & 60 & 0 & 8.6 & 1 \\
\hline Peroxiredoxin (Bcp) & 18 & 86 & 0 & 35 & 4 \\
\hline Glutaredoxin 4 & 13 & 55 & 0 & 19 & 2 \\
\hline
\end{tabular}

Cells expressing YchF with the UV-activated cross-linker pBpa at position 20 of YchF were grown on LB medium and one half was UV exposed (+UV), while the other half served as control (-UV). YchF was purified from both samples and separated by sodium dodecyl sulfate-polyacrylamide gel electrophoresis. Equal gel slices were cut out from both lanes, followed by in-gel digestion using trypsin and mass spectrometric analysis. Shown are data obtained for Escherichia coli proteins involved in antioxidant response.

${ }^{\text {a }}$ Protein identified.

${ }^{\mathrm{b}}$ Calculated molecular mass.

${ }^{\mathrm{c}}$ Molecular mass of cross-linking product determined by extrapolation.

${ }^{\mathrm{d}}$ Relative intensity observed in gel slices from the control lane (-UV) compared with the +UV lane.

${ }^{\text {e}} \mathrm{Sequence}$ coverage of total sequence by detected peptides.

${ }^{\mathrm{f}}$ Number of unique peptides detected. 

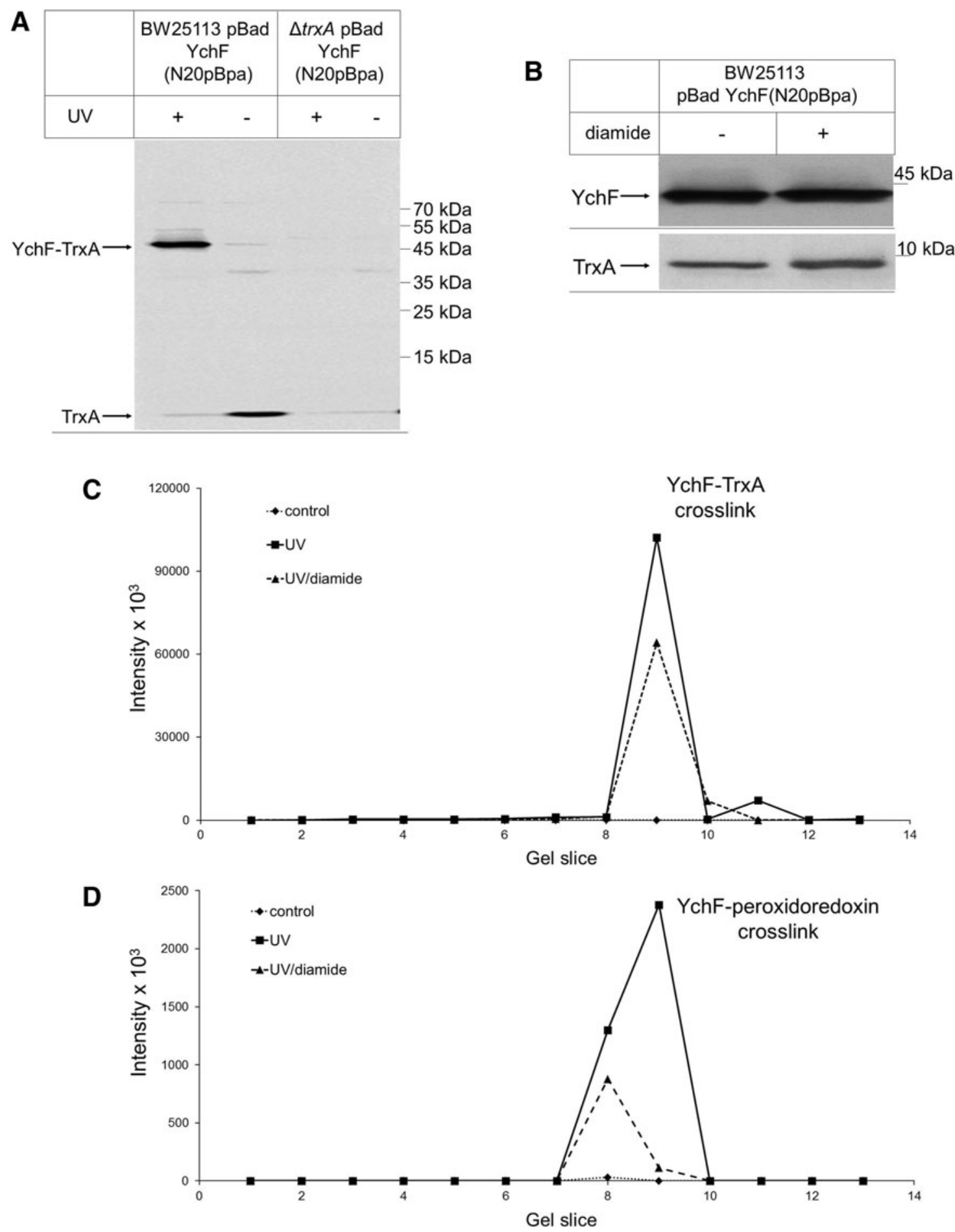

FIG. 5. YchF cross-links to thioredoxin in vivo. (A) Wild-type E. coli cells or a $\Delta \operatorname{trx} A$ E. coli strain expressing YchF with the UV-dependent cross-linker pBpA incorporated at position N20 were either UV exposed (+) or kept in the dark (-). After cross-linking, YchF was purified, separated by SDS-PAGE, and after Western transfer probed with $\alpha$-TrxA antibodies. (B) Wild-type E. coli cells expressing $\mathrm{YchF}(\mathrm{N} 20 \mathrm{pBpa})$ were treated with $50 \mu M$ diamide and TCA precipitated. The pellet after centrifugation was separated by SDS-PAGE and after Western transfer probed with either $\alpha$-YchF or with $\alpha$-TrxA antibodies, (C, D) YchF(N20pBpa) cross-linking products were separated by SDS-PAGE, individual gel slices corresponding to the MW range of 130 to $40 \mathrm{kDa}$ were excised, subjected to in-gel digestion using trypsin, and analyzed by mass spectrometry. Data were analyzed for peptides derived from TrxA (C) and peroxiredoxin (D). Shown are summed peptide intensities measured in samples obtained without UV exposure (noncross-linked, $\bullet$, dotted line), after UV exposure (crosslinked, $\mathbf{\square}$, solid line), and after UV exposure of diamide-treated cells ( $\mathbf{\Lambda}$, dashed line). 


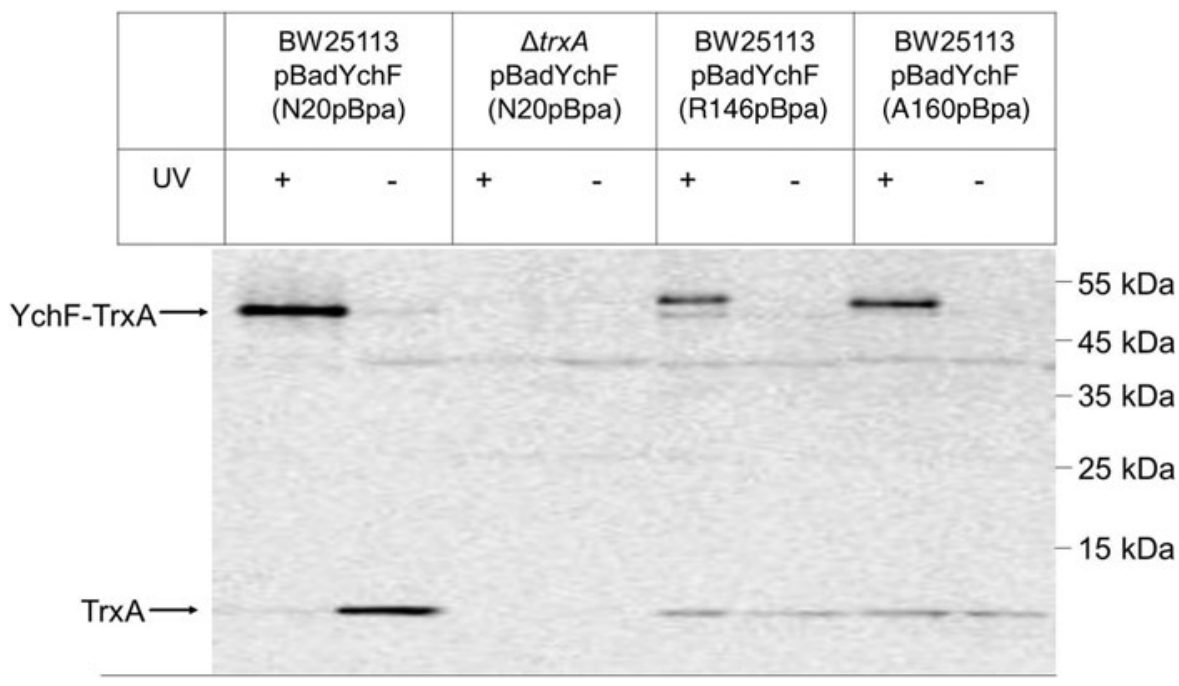

FIG. 6. Thioredoxin interacts with both the $\mathrm{N}$-terminus and the helical domain of YchF in vivo. In vivo cross-linking was performed as in Fig. 5 with pBpa inserted at position N20, R146, or N160. Samples were subsequently analyzed by Western blotting using $\alpha$-TrxA antibodies. Cross-linked products of positions, R146 and 160, migrated in two distinct species, which is a common phenomenon of $\mathrm{pBpa}$ cross-linking $(25,31,38)$ and probably reflects different threedimensional structures. presence of equimolar amounts of TrxA, we observed about two-fold stimulation of the YchF ATPase activity, which was comparable with the stimulatory effect of DTT (Fig. 7A). The stepwise increase of the TrxA concentration to a YchF:TrxA ratio of 1:4 did not significantly stimulate the ATPase activity further (Fig. 7A). These data demonstrate that the YchFTrxA interaction accelerates the ATPase activity of YchF and establish the physiological significance of the YchF-TrxA interaction observed via in vivo cross-linking.

We also tested whether TrxA would dissociate the YchF dimer. When purified YchF was separated under nonreducing conditions by SDS-PAGE, we observed both the YchF monomer and dimer by Western blotting (Fig. 7B). However, when YchF was incubated with TrxA before SDS-PAGE and Western blotting, the YchF dimer was almost undetectable. As a control, we added TrxA to YchF without further incubation and separated it directly on SDS-PAGE. Even without additional incubation, TrxA significantly reduced the YchF dimer. Thus, TrxA dissociates the YchF dimer, which concomitantly stimulates the ATPase activity of YchF.

\section{Discussion}

The YchF/Ola1 subfamily constitutes one of the most conserved members of the P-Loop GTPase family and is characterized by its unique preference for ATP $(24,52)$. Sequence comparison between $E$. coli $\mathrm{YchF}$ and human hOla1 revealed $45 \%$ sequence identity and $62 \%$ sequence similarity, but there is so far no clear consensus about the possible role of this protein family. Disclosing cellular targets of YchF/Ola1 is of significant importance because human Ola1 appears to play a major role in pathogenicity and disease development. Ola1 expression is regulated by DNA damage $(32,46)$ and is upregulated in many human tumors (46). On the other hand, a downregulation of Ola1 is observed in interferon $\beta$-treated multiple sclerosis patients (14). Furthermore, cell proliferation and apoptosis seem to be influenced by Ola1 (23). In both bacteria and humans, overexpression of YchF/Ola1 increases the sensitivity toward oxidative stress $(53,56,57)$ and an imbalance of the oxidative stress response could potentially provide the link between DNA damage repair (40), the pathogenesis of multiple sclerosis (30), and apoptosis (43).
The YchF/Ola1 mode of action is unknown, but the available data support a role as inhibitor of the oxidative stress response by a transcription- and translation-independent mechanism $(53,56)$. E. coli cells expressing ATPase-deficient YchF derivatives do not display $\mathrm{H}_{2} \mathrm{O}_{2}$ hypersensitivity (53). This suggests that the ATPase activity of YchF is required for inhibition of the oxidative stress response, probably by regulating the interaction of $\mathrm{YchF}$ with proteins such as KatG or the iron scavenger Dps $(19,53)$. The ATPase activity is subject to a complex regulatory regime (53) and our data now demonstrate that $\mathrm{YchF}$ undergoes a reversible redox-sensitive dimerization, which inactivates its ATPase activity. Dimer formation depends on Cys35, which is conserved from $E$. coli to humans and located next to the conserved threonine residue within the G2 motif of the ATPase domain (Fig. 1). This provides the explanation for the ATPase-inhibiting effect of YchF dimerization. The G2 motif serves as the effector region of GTPases, and hydrogen bonds between the threonine residue and the $\gamma$-phosphate are required for catalysis and the subsequent conformational changes (54). This so-called loaded spring mechanism is most likely impaired upon disulfide bond formation of the nearby cysteine residue.

TrxA or reducing agents such as DTT dissociate the YchF dimer and activate its full ATPase activity. Thus, the redoxregulated monomer-dimer equilibrium of YchF appears to be a key event in the functional cycle of YchF. Wild-type E. coli cells produce $\sim 15 \mu M \mathrm{H}_{2} \mathrm{O}_{2} / \mathrm{s}$ (41), which is too low for significant thiol oxidation (19). Still, we observed YchF dimer formation in $E$. coli cells without $\mathrm{H}_{2} \mathrm{O}_{2}$ treatment. This could indicate that the active site thiols of $\mathrm{YchF}$ are unusually reactive, which has also been observed for OxyR and AhpCF (4). In addition, the detection of YchF in E. coli cells by Western blotting required moderate overexpression. Assuming that YchF inhibits the antioxidant response, the endogenous $\mathrm{H}_{2} \mathrm{O}_{2}$ concentration in YchF-overexpressing cells might actually be higher than in wild-type $E$. coli cells, which would promote the formation and stability of the YchF dimer even in the absence of exogenous $\mathrm{H}_{2} \mathrm{O}_{2}$. Our current model suggests that under native conditions, YchF exists predominantly as a monomer due to the reducing conditions in the bacterial cytosol. This would allow for the full ATPase activity, which is required for inactivation of antioxidant 
FIG. 7. Thioredoxin increases the ATPase activity of YchF and reduces the YchF dimer. (A) Purified $\mathrm{YchF}(0.2 \mu M)$ was incubated with either DTT $(10 \mathrm{mM})$ (gray bar) or increasing TrxA concentrations (hatched bars) and the ATPase activity was measured as in Fig. 4. The activity of wildtype $\mathrm{YchF}$ was set to one and corresponds to $0.35 \mathrm{nmol} \mathrm{ATP} /(\min \times$ $\mathrm{mg}$ protein). The values observed were corrected for the background ATP hydrolysis detected in the purified TrxA sample. (B) Purified YchF was incubated for $30 \mathrm{~min}$ at $37^{\circ} \mathrm{C}$ in the presence or absence of purified TrxA in a 1:1 molar ratio. As a control, TrxA was added to YchF without further incubation (w/o incubation). Samples were analyzed by SDS-PAGE under nonreducing conditions and probed with $\alpha$-YchF antibodies after Western transfer.
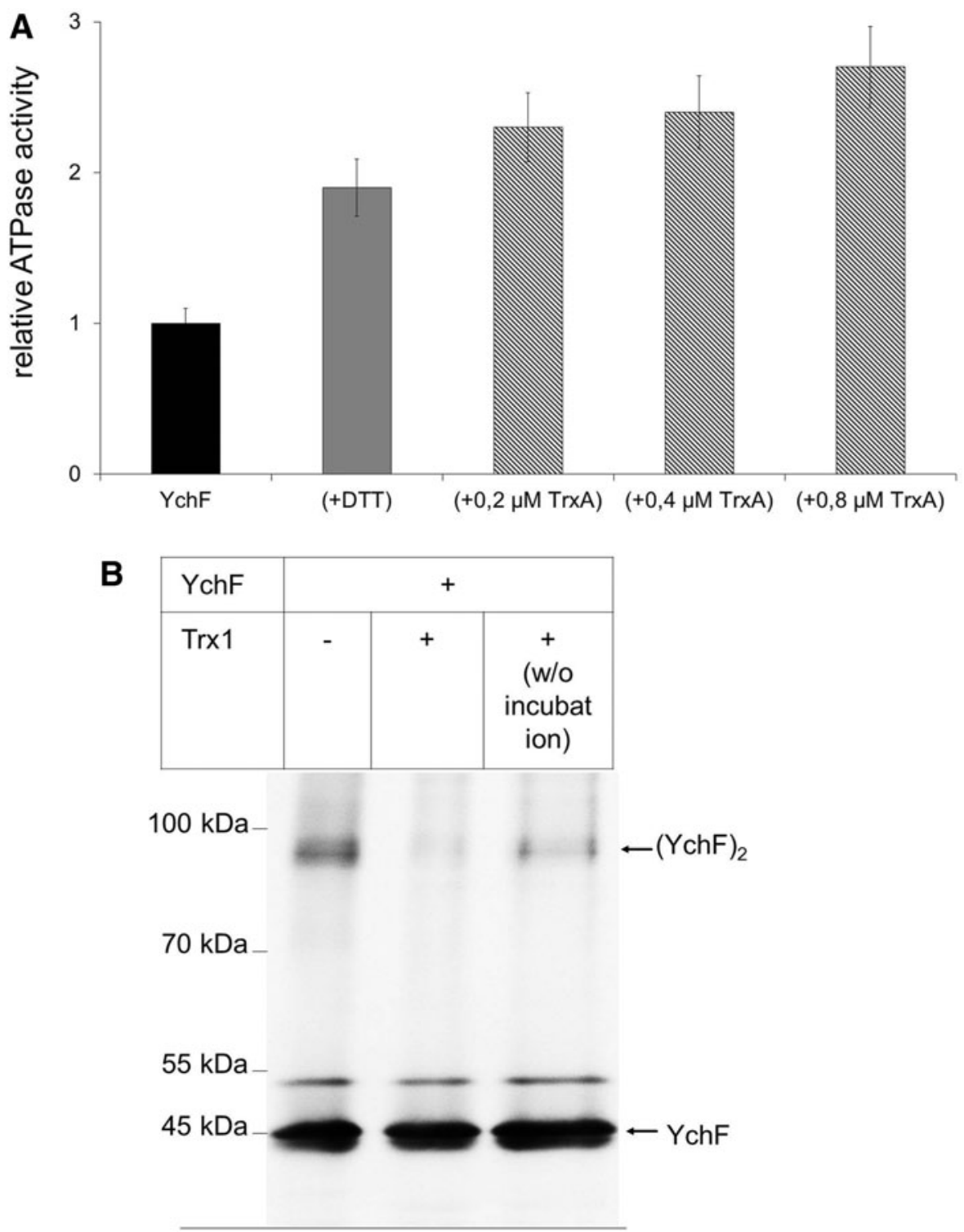

enzymes. In the presence of oxidative stress, YchF dimerization would prevent ATP hydrolysis and the inhibitory effect of YchF on antioxidant enzymes diminishes (Fig. 8). Redox-dependent dimerization is a well-known effect in biology and has been observed in response to oxidative stress for peroxiredoxins (5), mitofusins (42), or the redox chaperone, Hsp33 (18). Dimer dissociation of peroxiredoxin and Hsp33 is facilitated by the thioredoxin and glutaredoxin systems $(18,27)$, similar to what we observe for YchF. Intriguingly, our data identified TrxA as the predominant redox partner of YchF. In contrast to TrxC, TrxA in E. coli is not under OxyR control $(59,60)$ and thus not upregulated in the presence of oxidative stress. This probably reduces YchF monomerization under oxidative stress conditions.

In YchF-overexpressing cells, catalase activity is reduced (53) and the interaction of $\mathrm{YchF}$ with the three main $\mathrm{H}_{2} \mathrm{O}_{2}$ detoxifying enzymes, KatG, KatE, AhpCF, in E. coli has been demonstrated (Table 2) (53). Still, the exact mechanism of catalase inhibition remains to be identified. YchF could serve as a trap for these enzymes under nonstress conditions, allowing their immediate reactivation upon oxidative stress. This strategy could be part of the adaptive response against oxidative stress that has been observed in both bacteria and eukaryotes (20). The regulation of $\mathrm{H}_{2} \mathrm{O}_{2}$-degrading enzymes is also important for $\mathrm{H}_{2} \mathrm{O}_{2}$-dependent signaling processes, which stimulate cell proliferation and migration in eukaryotic cells (51). A role of Ola1/YchF in regulating the endogenous $\mathrm{H}_{2} \mathrm{O}_{2}$ concentration is supported by several observations. Knockdown of Ola1 has been shown to influence the migration of cancer cells, probably by modulation of the intracellular ROS levels (57). In addition, focal adhesion kinase is downregulated in $\mathrm{H}_{2} \mathrm{O}_{2}$-treated mesenchymal stem cells (44), but upregulated upon Ola1 knockdown (22), which is expected if Ola1 inhibits $\mathrm{H}_{2} \mathrm{O}_{2}$-degrading enzymes. The existence of $\mathrm{H}_{2} \mathrm{O}_{2}$-dependent signaling processes in bacteria has not been clearly demonstrated, but the catalytic mechanism of catalases might be a further reason for their inhibition in the absence of oxidative stress. At low $\mathrm{H}_{2} \mathrm{O}_{2}$ concentrations, the two-step catalytic cycle of catalases can stall with the heme cofactor in its ferryl intermediate state (36), which 


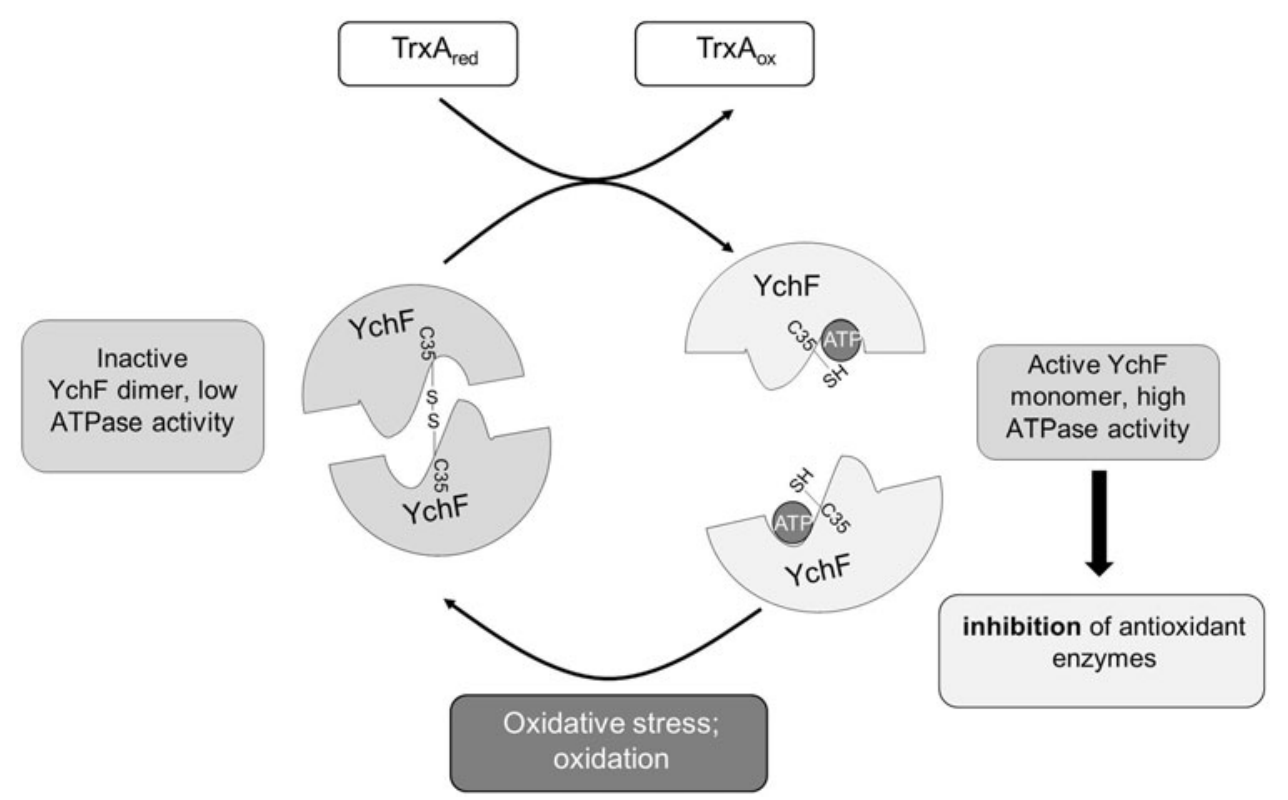

FIG. 8. Model for the redox regulation of YchF, a universally conserved inhibitor of the oxidative stress response. YchF acts as inhibitor of the oxidative stress response in both E. coli (53) and H. sapiens $(56,57)$. Oxidative stress regulates YchF expression and activity at both the transcriptional level (53) and the post-translational level. In the absence of oxidative stress, YchF exists primarily as a monomer with high ATPase activity, which is required for the inhibition of antioxidant enzymes. Upon oxidative stress, cysteine residue 35, which is located within the G2 motif of the ATPase domain, is oxidized, leading to YchF dimer formation, which prevents ATP hydrolysis. YchF dimerization upon oxidative stress probably includes an intramolecular disulfide bridge between cysteine 5 and cysteine 35 as an intermediate. Dimerization and concomitant ATPase inhibition alleviate the inhibition of antioxidant enzymes. If oxidative stress eases, YchF dimers are dissociated by the activity of thioredoxin 1 (TrxA).

will then abstract electrons from the surrounding polypeptide and thus function as a damaging oxidant. In addition, catalase peroxidases also efficiently oxidize $\mathrm{NAD}(\mathrm{P}) \mathrm{H}$, which is linked to superoxide radical formation (19). Thus, the posttranslational inhibition of antioxidant enzymes in the absence of oxidative stress is probably important for both unicellular and multicellular organisms.

The interaction between $\mathrm{YchF}$ and TrxA probably adds to the multiple effects that are associated with YchF/Ola1 overexpression because this will likely reduce the concentration of thioredoxin available for other cellular processes. In eukaryotic cells, the thioredoxin-interacting protein, Txnip, has been shown to be a key element of cellular redox regulation because it inhibits thioredoxin activity (34). Txnip promotes apoptosis, increases ROS production, and influences metastasis (55), phenotypes which have also been associated with increased Ola1 concentrations $(23,46,56)$.

Finally, the involvement of YchF/Ola1 in regulating oxidative stress could also explain why these proteins hydrolyze ATP rather than GTP. Guanine is particularly sensitive to oxidation due to its low redox potential (17), and the product of guanine oxidation, 7,8-dihydro-8-oxoguanine, is routinely determined for monitoring oxidative DNA damage (21). ATP has a significantly higher redox potential compared with GTP (48) and is less sensitive toward oxidation. Furthermore, upon oxidative stress, the ATP level transiently increases, probably due to the inhibition of energy-consuming processes (1). Using ATP rather than GTP could therefore reflect the functional adaptation of the Ola1/YchF subfamily to regulation of the oxidative stress response.

\section{Materials and Methods}

\section{Bacterial strains and growth conditions}

E. coli strains, BW25113, MRE600, BL21, and BL21(DE3), were used as wild-type strains and were grown in LB medium at $37^{\circ} \mathrm{C} ; 25 \mu \mathrm{g} / \mathrm{ml}$ kanamycin was added for the growth of JW1194 $(\Delta y c h F)$ and MY1013 ( $\Delta t r x A) ; 50 \mu \mathrm{g} / \mathrm{ml}$ ampicillin was added for strains carrying pBad-ychF or its derivatives; and $35 \mu \mathrm{g} / \mathrm{ml}$ chloramphenicol for strains carrying pSupBpaRS-6TRN (37). Plasmid construction and mutagenesis are described in Supplementary Data. Spot assays and inhibition assays in the presence of diamide were performed as described for $\mathrm{H}_{2} \mathrm{O}_{2}(53)$.

\section{Protein purification and ATPase assay}

To purify N-terminally His-tagged thioredoxin 1, BL21carrying pET19b-trxA was grown to an optical density (OD) of 0.5 , and then induced by $1 \mathrm{~m} M$ IPTG. After $3 \mathrm{~h}$, cells were harvested, resuspended in EPTX buffer ( $25 \mathrm{~m} M$ HEPES $\mathrm{KOH}, \mathrm{pH} 7.5,1 \mathrm{M} \mathrm{NH}_{4}$-acetate, $0.4 \mathrm{~m} M \mathrm{Mg}$-acetate), and stored at $-20^{\circ} \mathrm{C}$. After thawing, Complete protease inhibitor mixture (Roche) and phenylmethylsulfonyl fluoride (PMSF, final concentration $0.5 \mathrm{mM}$ ) were added. Cells were lysed thrice by a French pressure cell (Thermo Scientific). Cell debris was removed by centrifugation $(30 \mathrm{~min}, 15,500 \mathrm{rpm}$, Sorvall SS-34 rotor). The supernatant was centrifuged for $1 \mathrm{~h}$ at 50,000 rpm (Beckmann Ti50 rotor). The supernatant was mixed with EPTX $(+5 \mathrm{~m} M$ imidazole)-equilibrated Talon beads (Clontech) and incubated at $4^{\circ} \mathrm{C}$ for $2 \mathrm{~h}$. The Talon 
beads were washed five times with $3 \mathrm{ml} 5 \mathrm{mM}$ imidazole in EPTX buffer and proteins were eluted with an imidazole gradient (100-300 $\mathrm{mM}$ ) in EPTX buffer. Eluted proteins were buffer exchanged to $1 \times$ HT buffer $(50 \mathrm{~m} M$ Hepes- $\mathrm{KOH}$, pH 7.6; $100 \mathrm{~m} M \mathrm{~K}$-acetate, $10 \mathrm{~m} M \mathrm{Mg}$-acetate) using PD10 columns (GE Healthcare).

Purification of YchF carrying a C-terminal His-tag followed the same protocol, but cells were induced with $0.001 \%$ arabinose. Cysteine-free YchF was purified from inclusion bodies after the first centrifugation step. The pellet was resuspended in $6 \mathrm{M}$ urea in EPTX buffer at $4^{\circ} \mathrm{C}$ for $5 \mathrm{~h}$. After centrifugation $(15,500 \mathrm{rpm}, 30 \mathrm{~min}$, Sorvall SS-34 rotor) the supernatant was incubated with EPTX-equilibrated Talon beads and further purified as described above. ATPase activity of YchF was determined by measuring hydrolysis of $\gamma$ - ${ }^{33}$ P-labeled ATP (53).

\section{Cell disruption with lysozyme}

E. coli cells were grown to an OD of 0.5 and YchF expression was induced by $0.001 \%$ arabinose. Two hours after induction, cells were harvested and washed twice with Tris$\mathrm{HCl}$ ( $\mathrm{pH}$ 8.0). After centrifugation, the cells were resuspended in Tris- $\mathrm{HCl}$ (pH 8.0), $1 \mathrm{~m} M$ EDTA, $25 \mathrm{mM} \mathrm{NaCl}$ containing lysozyme (Sigma) $(10 \mathrm{mg} / \mathrm{ml})$, Complete protease inhibitor (Roche), and $0.5 \mathrm{mM}$ PMSF. The suspension was incubated for $30 \mathrm{~min}$ at $37^{\circ} \mathrm{C}$. Subsequently, the cells were frozen five times in liquid nitrogen and thawed in water. The suspension was centrifuged $(14,000 \mathrm{rpm}, 10 \mathrm{~min})$ and the supernatant was loaded onto an SDS-PAGE.

\section{Sodium dodecyl sulfate-polyacrylamide gel electrophoresis}

Samples were denatured at $37^{\circ} \mathrm{C}$ for $10 \mathrm{~min}$. Samples for nonreducing SDS-PAGE were resuspended in DTT-free 4x Laemmli loading buffer (278 $\mathrm{m} M$ Tris- $\mathrm{HCl}, \mathrm{pH}$ 6.8, 44.4\% glycerol, $4.4 \%$ SDS, $0.02 \%$ bromophenol blue). Reducing loading buffer contained fresh DTT at a final concentration of $25 \mathrm{~m} M$.

For two-dimensional SDS-PAGE, purified YchF was separated by nonreducing SDS-PAGE as the first dimension. The gel was stained with colloidal Coomassie and the YchF monomer and dimer bands were excised and incubated in $200 \mu \mathrm{l}$ of denaturing buffer $(50 \mathrm{~m} M$ Tris- $\mathrm{HCl} \mathrm{pH} 6.8,30 \%$ glycerol, $2 \% \mathrm{SDS}, 6 \mathrm{M}$ urea, $1 M \mathrm{DTT}$ ) for $30 \mathrm{~min}$ at $54^{\circ} \mathrm{C}$. The sample was separated by reducing SDS-PAGE as the second dimension and stained with colloidal Coomassie.

\section{Western blot analyses}

For Western blot analyses, the proteins were blotted onto nitrocellulose membranes (GE Healthcare). $\alpha$-YchF antibodies were raised in rabbits against the peptide VNEDGFENNPYLDQC. $\alpha$-TrxA antibodies were obtained from Sigma. A horseradish peroxidase-coupled secondary antibody was used for detection; blots were incubated for $1 \mathrm{~min}$ with homemade enhanced chemiluminescence reagent and signals were detected by a CCD camera.

\section{In vivo site-directed cross-linking}

E. coli cells carrying pSUP-BpaRS-6TRN and pBadYchFN20, pBad-YchFR146, or pBad-YchFA160 were grown overnight and used for the inoculation of $400 \mathrm{ml} \mathrm{LB}$; $0.5 \mathrm{~m} M \mathrm{pBpA}$ in $1 M \mathrm{NaOH}$ was added and cells were grown at $37^{\circ} \mathrm{C}$ to an OD of 0.3 before they were induced by $0.001 \%$ arabinose. Cells were harvested after $3 \mathrm{~h}$ of growth and resuspended in $8 \mathrm{ml}$ PBS buffer. Half of the sample was transferred into a six-well microtiter plate and treated with UV light $\left(0.12 \mathrm{~J} / \mathrm{cm}^{2}\right.$ ) (UV Transilluminator Vilber Lourmat BLX-365) for $20 \mathrm{~min}$. The other half was protected against UV light. YchF was then purified from UV-exposed and control cells following the protocol described above. To analyze the effect of thiol depletion on in vivo cross-linking, cells were treated for $30 \mathrm{~min}$ with $50 \mu M$ diamide (Sigma) at $37^{\circ} \mathrm{C}$ before harvesting.

\section{Protein identification and quantification by mass spectrometry}

Lanes of an SDS gel of purified samples were cut into equal slices and subjected to in-gel digestion using trypsin, followed by nano-UHPLC/MS analysis essentially as described (38). The LTQ-FT-Ultra (Thermo Fisher Scientific) was operated with settings as described (33), except that survey full MS spectra were collected at a resolution of 25,000 with an automatic gain control target value of $2 \times 10^{6}$ ions. Proteins were identified by database searches using the MaxQuant program version 1.3.0.5 $(12,13)$ as described $(33)$, selecting carbamidomethylation of cysteine as a fixed modification. Protein sequences were taken from the UniProt database (50) for E. coli (version 2013_07, taxonomy: 83333, keywords: 181 and 1185). Intensities per protein (representing the sum of unique peptide intensities) and per gel slice were used to generate profiles for individual gel lanes and to estimate the molecular mass of crosslink products by extrapolation. Relative quantification was done by dividing the total intensity per protein observed in the control lane (-UV) by that observed in the +UV lane.

\section{Identification of disulfide bonds by mass spectrometry}

To block free cysteine residues, purified YchF $(3.5 \mathrm{mg})$ was treated with $100 \mathrm{~m} M$ iodoacetamide for $15 \mathrm{~min}$ at $37^{\circ} \mathrm{C}$ before separation by SDS-PAGE. After colloidal Coomassie staining, the monomer and the dimer bands were excised. Gel slices were washed and destained by five successive alternate 10-min incubations with $10 \mathrm{~m} M \mathrm{NH}_{4} \mathrm{HCO}_{3}$, followed by $50 \%$ ethanol in $10 \mathrm{mM} \mathrm{NH} \mathrm{NHCO}_{3}$. To minimize disulfide scrambling, the $\mathrm{pH}$ was adjusted to 6.5 with acetic acid. Furthermore, $2 \mathrm{~m} M$ N-ethylmaleimide (NEM) was included in both solutions to prevent the formation of non-native disulfide bonds. In-gel protein digestion using trypsin was carried out at $\mathrm{pH} 6.5$ in $10 \mathrm{~m} M \mathrm{NH}_{4} \mathrm{HCO}_{3}$ supplemented with $2 \mathrm{~m} M$ NEM. Samples were incubated with $66 \mathrm{ng}$ trypsin (sequencing grade modified, Promega) per gel slice for $15 \mathrm{~h}$ at $37^{\circ} \mathrm{C}$. Peptides were eluted in $0.05 \%$ trifluoroacetic acid (TFA) $/ 50 \%$ acetonitrile (ACN), dried in vacuum, and redissolved in $15 \mu \mathrm{l} 0.1 \%$ TFA. Samples were analyzed by nanoHPLC/ESI-MS/MS as described (33), except that peptides were separated on a $50 \mathrm{~cm} \times 70 \mu \mathrm{m} \mathrm{C} 18$ column (Acclaim PepMap RSLC column, $2 \mu \mathrm{m}$ particle size, $100 \AA$ pore size; Thermo Scientific) at a flow rate of $250 \mathrm{nl} / \mathrm{min}$ with a linear gradient consisting of $1 \%$ to $65 \%$ solvent $\mathrm{B}(0.1 \%$ formic acid, $4 \%$ dimethylsulfoxide, $30 \%$ methanol, $48 \%$ ACN) for $30 \mathrm{~min}$. The identification of disulfide-linked peptides is described in Supplementary Data. 


\section{Cysteine labeling with PEG-Mal}

Purified YchF in $50 \mathrm{~m} M$ Tris- $\mathrm{HCl}, \mathrm{pH} 7.5,5 \mathrm{mM} \mathrm{MgCl} 2$ and $200 \mathrm{~m} M \mathrm{NaCl}$ was reduced by incubating it for $1 \mathrm{~h}$ at $4^{\circ} \mathrm{C}$ with $150 \mu \mathrm{l}$ Thermo Scientific ${ }^{\mathrm{TM}}$ Immobilized TCEP Disulfide Reducing Gel and subsequently incubated with $0.75 \mathrm{~m} M$ PEG-MAL (in DMSO) for $15 \mathrm{~min}$ at $25^{\circ} \mathrm{C}$. Loading buffer (-DTT) was added and the samples were denatured at $37^{\circ} \mathrm{C}$ for $10 \mathrm{~min}$ and loaded onto SDS-PAGE.

\section{Cysteine labeling with fluorescein-5-maleimide}

Purified YchF $(5 \mathrm{mg})$ was reduced by incubation with TCEP Reducing Gel for $2 \mathrm{~h}$ at $4^{\circ} \mathrm{C}$ and continuous shaking (750 rpm). After centrifugation $(21,000 \mathrm{~g}, 3 \mathrm{~min}), \sim 50 \mu \mathrm{g}$ of fluorescein-5-maleimide (in HT buffer) was added to the supernatant, followed by a 2 -h incubation at room temperature. Precipitates were removed by centrifugation $(21,000 \mathrm{~g}$, $1 \mathrm{~min}$ ) and both supernatant and pellet were denatured for $10 \mathrm{~min}$ at $54^{\circ} \mathrm{C}$ in Laemmli loading buffer (-DTT). After SDS-PAGE, fluorescence was detected using a CCD camera.

\section{Acknowledgments}

This work was supported by grants from the Deutsche Forschungsgemeinschaft to B.W. and H.G.K., the Excellence Initiative of the German Federal and State Governments (Grant EXC 294 BIOSS Centre for Biological Signalling Studies) to B.W., the Else-Kröner-Fresenius Stiftung and the Motivate MD College of the University Freiburg Medical School to L.H., and the Chinese Scholarship Council to Q.B. The authors thank the National Institute of Genetics, Japan, for E. coli strains, JW1194 ( $\Delta y c h F)$ and MY1013 ( $\Delta$ trxA).

\section{Author Disclosure Statement}

No competing financial interests exist.

\section{References}

1. Akhova AV and Tkachenko AG. ATP/ADP alteration as a sign of the oxidative stress development in Escherichia coli under antibiotic treatment. FEMS Microbiol Lett 353: 6976, 2014.

2. Alexander RW and Schimmel P. Domain-domain communication in aminoacyl-tRNA synthetases. Prog Nucleic Acid Res Mol Biol 69: 317-349, 2001.

3. Antelmann $\mathrm{H}$ and Helmann JD. Thiol-based redox switches and gene regulation. Antioxid Redox Signal 14: 1049-1063, 2011.

4. Aslund F, Zheng M, Beckwith J, and Storz G. Regulation of the OxyR transcription factor by hydrogen peroxide and the cellular thiol-disulfide status. Proc Natl Acad Sci U S A 96: 6161-6165, 1999.

5. Baker LM and Poole LB. Catalytic mechanism of thiol peroxidase from Escherichia coli. Sulfenic acid formation and overoxidation of essential CYS61. J Biol Chem 278: 9203-9211, 2003.

6. Becker M, Gzyl KE, Altamirano AM, Vuong A, Urban K, and Wieden HJ. The 70S ribosome modulates the ATPase activity of Escherichia coli YchF. RNA Biol 9: 1288-1301, 2012.

7. Charles R, Jayawardhana T, and Eaton P. Gel-based methods in redox proteomics. Biochim Biophys Acta 1840: 830-837, 2014.
8. Chen YC and Chung YT. A conserved GTPase YchF of Vibrio vulnificus is involved in macrophage cytotoxicity, iron acquisition, and mouse virulence. Int J Med Microbiol 301: 469-474, 2011.

9. Cheung MY, Li MW, Yung YL, Wen CQ, and Lam HM. The unconventional P-loop NTPase OsYchF1 and its regulator OsGAP1 play opposite roles in salinity stress tolerance. Plant Cell Environ 36: 2008-2020, 2013.

10. Cheung MY, Xue Y, Zhou L, Li MW, Sun SS, and Lam HM. An ancient P-loop GTPase in rice is regulated by a higher plant-specific regulatory protein. J Biol Chem 285: 37359-37369, 2010.

11. Choi H, Kim S, Mukhopadhyay P, Cho S, Woo J, Storz G, and Ryu SE. Structural basis of the redox switch in the OxyR transcription factor. Cell 105: 103-113, 2001.

12. Cox J and Mann M. MaxQuant enables high peptide identification rates, individualized p.p.b.-range mass accuracies and proteome-wide protein quantification. Nat Biotechnol 26: 1367-1372, 2008.

13. Cox J, Neuhauser N, Michalski A, Scheltema RA, Olsen JV, and Mann M. Andromeda: a peptide search engine integrated into the MaxQuant environment. J Proteome Res 10: 1794-1805, 2011.

14. Croze E, Yamaguchi KD, Knappertz V, Reder AT, and Salamon H. Interferon-beta- $1 b$-induced short- and longterm signatures of treatment activity in multiple sclerosis. Pharmacogenomics J 13: 443-451, 2013.

15. Danese I, Haine V, Delrue RM, Tibor A, Lestrate P, Stevaux O, Mertens P, Paquet JY, Godfroid J, De Bolle X, and Letesson JJ. The Ton system, an ABC transporter, and a universally conserved GTPase are involved in iron utilization by Brucella melitensis 16M. Infect Immun 72: 57835790, 2004.

16. Gradia DF, Rau K, Umaki AC, de Souza FS, Probst CM, Correa A, Holetz FB, Avila AR, Krieger MA, Goldenberg $\mathrm{S}$, and Fragoso SP. Characterization of a novel Obg-like ATPase in the protozoan Trypanosoma cruzi. Int J Parasitol 39: 49-58, 2009.

17. Haghdoost S, Sjölander L, Czene S, Harms-Ringdahl M. The nucleotide pool is a signficant target for oxidative stress. Free Radic Biol Med 41: 620-626, 2006.

18. Hoffmann JH, Linke K, Graf PC, Lilie H, and Jacob U. Identification of a redox-regulated chaperone network. Embo J 23: 160-168, 2004.

19. Imlay JA. Cellular defenses against superoxide and hydrogen peroxide. Annu Rev Biochem 77: 755-776, 2008.

20. Imlay JA. The molecular mechanisms and physiological consequences of oxidative stress: lessons from a model bacterium. Nat Rev Microbiol 11: 443-454, 2013.

21. Jacob KD, Noren-Hooten N, Trzeciak AR, and Evans MK. Marker of oxidant stress that are clinically relevant in aging and age-related disease. Mech Ageing Dev 134: 139-157, 2013.

22. Jeyabal PV, Rubio V, Chen H, Zhang J, and Shi ZZ. Regulation of cell-matrix adhesion by OLA1, the Obg-like ATPase 1. Biochem Biophys Res Commun 444: 568-574, 2014.

23. Kira $Y$ and Nishikawa M. The identification and characterization of a new GTP binding protein (Gbp45) involved in cell proliferation and death related to mitochondrial function. Cell Mol Biol Lett 13: 570-584, 2007.

24. Koller-Eichhorn R, Marquardt T, Gail R, Wittinghofer A, Kostrewa D, Kutay U, and Kambach C. Human OLA1 defines an ATPase subfamily in the Obg family of GTPbinding proteins. J Biol Chem 282: 19928-19937, 2007. 
25. Kuhn P, Weiche B, Sturm L, Sommer E, Drepper F, Warscheid B, Sourjik V, and Koch HG. The bacterial SRP receptor, SecA and the ribosome use overlapping binding sites on the SecY translocon. Traffic 12: 563-578, 2011.

26. Lee JW and Helmann JD. The PerR transcription factor senses $\mathrm{H} 2 \mathrm{O} 2$ by metal-catalysed histidine oxidation. Nature 440: 363-367, 2006.

27. Lu J and Holmgren A. The thioredoxin antioxidant system. Free Radic Biol Med 66: 75-87, 2014.

28. Macek B, Gnad F, Soufi B, Kumar C, Olsen JV, Mijakovic I, and Mann M. Phosphoproteome analysis of E. coli reveals evolutionary conservation of bacterial Ser/Thr/Tyr phosphorylation. Mol Cell Proteomics 7: 299-307, 2008.

29. Matsuzuwa A, Kanno S-i, Nakayama M, Mochiduki H, Wei L, Shimaoka T, Furukuwa Y, Kato K, Shibata S, Ishioka C, and Chiba N. The BRCA1/BRAD1-interacting protein Ola1 functions in centrosome Regulation. Mol Cell 53: 101-114, 2014.

30. Miller E, Wachnowicz B, and Majsterek I. Advances in oxidative therapy of multiple sclerosis. Curr Med Chem 20: 4720-4730, 2013.

31. Mori $\mathrm{H}$ and Ito K. Different modes of SecY-SecA interactions revealed by site-directed in vivo photo-crosslinking. Proc Natl Acad Sci U S A 103: 16159-16164, 2006.

32. Nabilsi NH, Broaddus RR, and Loose DS. DNA methylation inhibits p53-mediated survivin repression. Oncogene 28: 2046-2050, 2009.

33. Niemann M, Wiese S, Mani J, Chanfon A, Jackson C, Meisinger C, Warscheid B, and Schneider A. Mitochondrial outer membrane proteome of trypanosoma brucei reveals novel factors required to maintain mitochondrial morphology. Mol Cell Proteomics 12: 515-528, 2013.

34. Nishiyama A, Matsui K, Iwata S, Hirota K, Masutani H, Nakamura H, Takagi Y, Sono H, Gon Y, and Yodoi J. Identification of thioredoxin-binding protein-2/vitamin $\mathrm{D}(3)$ up-regulated protein 1 as a negative regulator of thioredoxin function and expression. J Biol Chem 274: 21645-21650, 1999.

35. Parry DAD, Fraser RDB, and Squire JM. Fifty years of coiled-coils and $\alpha$-helical bundles: A close relationship between sequence and structure. J Struct Biol 163: 258269, 2008.

36. Putnam CD, Arvai AS, Bourne Y, and Tainer JA. Active and inhibited catalase structures: ligand and NADPH binding and catalytic mechanism. J Mol Biol 296: 295-309, 2000.

37. Ryu Y and Schultz PG. Efficient incorporation of unnatural amino acids into proteins in Escherichia coli. Nat Methods 3: 263-265, 2006.

38. Sachelaru I, Petriman NA, Kudva R, Kuhn P, Welte T, Knapp B, Drepper F, Warscheid B, and Koch HG. YidC occupies the lateral gate of the SecYEG translocon and is sequentially displaced by a nascent membrane protein. $J$ Biol Chem 288: 16295-16307, 2013.

39. Samanfar B, Tan le H, Shostak K, Chalabian F, Wu Z, Alamgir M, Sunba N, Burnside D, Omidi K, Hooshyar M, Galvan Marquez I, Jessulat M, Smith ML, Babu M, Azizi A, and Golshani A. A global investigation of gene deletion strains that affect premature stop codon bypass in yeast, Saccharomyces cerevisiae. Mol Biosyst 10: 916-924, 2014.

40. Scott TL, Rangaswamy S, Wicker CA, and Izumi T. Repair of oxidative DNA damage and cancer: recent progress in
DNA base excision repair. Antioxid Redox Signal 20: 708726, 2014.

41. Seaver LC and Imlay JA. Are respiratory enzymes the primary sources of intracellular hydrogen peroxide? J Biol Chem 279: 48742-48750, 2004.

42. Shutt T, Geoffrion M, Milne R, and McBride HM. The intracellular redox state is a core determinant of mitochnodrial fusion. EMBO Rep 13: 909-915, 2012.

43. Sinha K, Das J, Pal PB, and Sil PC. Oxidative stress: the mitochondria-dependent and mitochondria-independent pathway of apoptosis. Arch Toxicol 87: 1157-1580, 2013.

44. Song H, Cha MJ, Song BW, Kim IK, Chang W, Lim S, Choi EJ, Ham O, Lee SY, Chung N, Jang Y, and Hwang KC. Reactive oxygen species inhibit adheasion of mesenchymal stemm cells implanted into ischemic myocardium via interference of focal adheasion complex. Stem Cells 31: 555-563, 2010.

45. Stebbins CE, Borukhov S, Orlova A, Polyakov A, Goldfarb A, and Darst SA. Crystal structure of the GreA transcript cleavage factor from Escherichia coli. Nature 373: 636640, 1995.

46. Sun H, Luo X, Montalbano J, Jin W, Shi J, Sheikh MS, and Huang Y. DOC45, a novel DNA damage-regulated nucleocytoplasmic ATPase that is overexpressed in multiple human malignancies. Mol Cancer Res 8: 57-66, 2010.

47. Teplyakov A, Obmolova G, Chu SY, Toedt J, Eisenstein E, Howard AJ, and Gilliland GL. Crystal structure of the YchF protein reveals binding sites for GTP and nucleic acid. $J$ Bacteriol 185: 4031-4037, 2003.

48. Thapa B and Schlegel HB. Calculations of $\mathrm{pKa}$ 's and redox potentials of nucelobases with explicit waters and polarizable continuum solvation. J Phys Chem 119: 5134-5144, 2015.

49. Tomar SK, Kumar P, and Prakash B. Deciphering the catalytic machinery in a universally conserved ribosome binding ATPase YchF. Biochem Biophys Res Commun 408: 459-464, 2011.

50. UniProtConsortium. Activities at the Universal Protein Resource (UniProt). Nucleic Acids Res 42: D191, 2013.

51. Veal EA, Day AM, and Morgan BA. Hydrogen peroxide sensing and signaling. Mol Cell 26: 1-14, 2007.

52. Verstraeten N, Fauvert M, Versees W, and Michiels J. The universally conserved prokaryotic GTPases. Microbiol Mol Biol Rev 75: 507-542, 2011.

53. Wenk M, Ba Q, Erichsen V, MacInnes K, Wiese H, Warscheid B, and Koch HG. A universally conserved ATPase regulates the oxidative stress response in Escherichia coli. J Biol Chem 287: 43585-43598, 2012.

54. Wittinghofer $A$ and Vetter IR. Structure-function relationsships of the G-domain, a canonical switch motif. Ann Rev Biochem 80: 943-971, 2011.

55. Yoshikara E, Masaki S, Matsuo Y, Chen Z, Tian HF, and Yodoi J. Thioredoxin/Txnip: redoxisome, as a redox switch for the pathogenesis of diseases. Front Immunol 4: 514, 2014.

56. Zhang J, Rubio V, Lieberman MW, and Shi ZZ. OLA1, an Obg-like ATPase, suppresses antioxidant response via nontranscriptional mechanisms. Proc Natl Acad Sci U S A 106: 15356-15361, 2009.

57. Zhang JW, Rubio V, Zheng S, and Shi ZZ. Knockdown of OLA1, a regulator of oxidative stress response, inhibits motility and invasion of breast cancer cells. $J$ Zhejiang Univ Sci B 10: 796-804, 2009. 
58. Zheng M, Aslund F, and Storz G. Activation of the OxyR transcription factor by reversible disulfide bond formation. Science 279: 1718-1721, 1998.

59. Zheng M, Wang X, Doan B, Lewis KA, Schneider TD, and Storz G. Computation-directed identification of OxyR DNA binding sites in Escherichia coli. J Bacteriol 183: 45714579, 2001.

60. Zheng M, Wang X, Templeton LJ, Smulski DR, LaRossa RA, and Storz G. DNA microarray-mediated transcriptional profiling of the Escherichia coli response to hydrogen peroxide. J Bacteriol 183: 4562-4570, 2001.

Address correspondence to: Prof. Hans-Georg Koch Institut für Biochemie und Molekularbiologie Albert-Ludwigs-Universität Freiburg Stefan-Meier Strasse 17 Freiburg 79104

Germany

E-mail: hans-georg.koch@biochemie.uni-freiburg.de
Date of first submission to ARS Central, January 28, 2015; date of final revised submission, June 22, 2015; date of acceptance, July 9, 2015.

$\begin{aligned} & \text { Abbreviations Used } \\ \text { BRCA1 } & =\text { breast cancer-associated gene } 1 \\ \text { DTT } & =\text { dithiothreitol } \\ \text { EDTA } & =\text { ethylenediaminetetraacetic acid } \\ \text { FDR } & =\text { false discovery rate } \\ \text { IPTG } & =\text { isopropyl } \beta \text {-D-1-thiogalactopyranoside } \\ \text { Obg }= & \text { spo0B-associated GTP-binding protein } \\ \text { OD } & =\text { optical density } \\ \text { pBpa } & =\text { para-benzoyl-L-phenylalanin } \\ \text { PBS } & =\text { phosphate-buffered saline } \\ \text { PEG-Mal } & =\text { methoxypolyethylene glycol maleimide } \\ \text { PMSF } & =\text { phenylmethylsulfonyl fluoride } \\ \text { SDS-PAGE }= & \text { sodium dodecyl sulfate-polyacrylamide gel } \\ & \text { electrophoresis } \\ \text { TCEP }= & \text { tris }(2-\text { carboxyethyl }) \text { phosphine } \\ \text { TGS-domain }= & \text { ThrRS-GTPases-SpoT } \\ \text { TRAFAC }= & \text { translation factor }\end{aligned}$

